\title{
NUMERICAL METHODS FOR SYSTEMS OF NONLINEAR INTEGRO-PARABOLIC EQUATIONS OF VOLTERRA TYPE
}

\author{
IGOR BOGLAEV
}

Communicated by Terry Herdman

\begin{abstract}
This paper deals with the numerical solution of systems of nonlinear integro-parabolic problems of Volterra type. The numerical approach is based on the method of upper and lower solutions. A monotone iterative method is constructed. Existence and uniqueness of a solution to the nonlinear difference scheme are established. An analysis of convergence rates of the monotone iterative method is given. Construction of initial upper and lower solutions is discussed. Numerical experiments are presented.
\end{abstract}

1. Introduction. Various reaction-diffusion-convection-type problems in the chemical, physical and engineering sciences are described by coupled systems of nonlinear integro-parabolic equations. In this paper, we give a numerical treatment for a coupled system of two nonlinear integro-parabolic equations of Volterra type. The integro-parabolic system under consideration is given in the form

$$
\begin{gathered}
\frac{\partial u_{i}}{\partial t}-L_{i} u_{i}+f_{i}(x, t, u)+\int_{0}^{t} g_{i}^{*}(x, t, s, u(x, s)) d s=0, \\
(x, t) \in \omega \times(0, T], \\
u_{i}(x, t)=\phi_{i}(x, t), \quad(x, t) \in \partial \omega \times(0, T], \\
u_{i}(x, 0)=\psi_{i}(x), \quad x \in \bar{\omega}, \quad i=1,2,
\end{gathered}
$$

where $u=\left(u_{1}, u_{2}\right), \omega$ is a connected bounded domain in $\mathbb{R}^{\kappa}, \kappa=$ $1,2, \ldots$, with boundary $\partial \omega$. The differential operators $L_{i}, i=1,2$, are

2010 AMS Mathematics subject classification. Primary 65M06, 65M12, 65M22, $65 \mathrm{R} 20$.

Keywords and phrases. System of nonlinear integro-parabolic equations of Volterra type, nonlinear difference schemes, monotone iterative method, upper and lower solutions.

Received by the editors on January 11, 2016. 
given by

$$
L_{i} u_{i}=\sum_{\alpha=1}^{\kappa} \frac{\partial}{\partial x_{\alpha}}\left(D_{i}(x, t) \frac{\partial u_{i}}{\partial x_{\alpha}}\right)+\sum_{\alpha=1}^{\kappa} v_{i, \alpha}(x, t) \frac{\partial u_{i}}{\partial x_{\alpha}}, \quad i=1,2,
$$

where the coefficients of the differential operators are smooth and $D_{i}$, $i=1,2$, are positive in $\bar{\omega} \times[0, T]$. It is also assumed that the functions $f_{i}, g_{i}^{*}, \phi_{i}$ and $\psi_{i}, i=1,2$, are smooth in their respective domains.

Such problems arise in many applications, including reaction dynamics $[3,7,9]$, heat conduction in materials with memory [4], population dynamics [5], compression of poro-viscoelastic media [6], and diffusion phenomena with memory effects $[\mathbf{1 3}, \mathbf{1 5}]$.

To discretize problem (1.1), we use the implicit scheme for parabolic equations and approximate (1.1) by a nonlinear difference scheme. The purpose of this paper is to develop a monotone iterative method for solving the nonlinear difference scheme, including the existence and uniqueness of a discrete solution, and error estimates of the iterative method. Our iterative method is based on the method of upper and lower solutions and associated monotone iterates. By using upper and lower solutions as two initial iterations, one can construct two monotone sequences which converge monotonically from above and below, respectively, to a solution of the nonlinear difference scheme.

Monotone iterative schemes for solving systems of parabolic equations were used in $[\mathbf{8}, \mathbf{1 0}]$. Here, the two important points in investigating the monotone iterative methods concerning a stopping criterion on each time level and estimates of convergence rates were omitted. In [1], we extended the monotone iterative method from $[\mathbf{8}, \mathbf{1 0}]$ to the case when, on each time level, a nonlinear difference scheme is solved inexactly, and gave an analysis of convergence rates of the monotone iterative method.

In [2], we constructed and investigated the monotone iterative method for solving a scalar nonlinear integro-parabolic problem of Volterra type in the case when, on each time level, a nonlinear difference scheme is solved inexactly, and gave an analysis of convergence rates of the monotone iterative method. In this paper, we extend the monotone iterative method from [2] to the case of the system of integro-parabolic problems of Volterra type (1.1). 
The structure of the paper as follows. In Section 2, we introduce a nonlinear difference scheme for the numerical solution of (1.1). A monotone iterative method is presented in Section 3. Existence and uniqueness of the solution to the nonlinear difference scheme are established. An analysis of convergence rates of the monotone iterative method is given. Convergence of the nonlinear difference scheme to the nonlinear integro-parabolic problem (1.1) is established. Section 4 deals with construction of initial upper and lower solutions. Finally, Section 5 presents results of numerical experiments.

2. The nonlinear difference scheme. On the domains $\bar{\omega}$ and $[0, T]$, we introduce meshes $\bar{\omega}^{h}$ and $\bar{\omega}^{\tau}$, respectively. The integrals in (1.1) are approximated by the finite sums $g_{i}, i=1,2$, based on the Riemann sum (the rectangular rule) [14]

$$
g_{i}\left(p, t_{k}, U\right)=\sum_{l=1}^{k} \tau_{l} g_{i}^{*}\left(p, t_{k}, t_{l}, U\left(p, t_{l}\right)\right), \quad\left(p, t_{k}\right) \in \bar{\omega}^{h} \times \omega^{\tau},
$$

where $\omega^{\tau} \equiv \bar{\omega}^{\tau} \backslash\{0\}, U=\left(U_{1}, U_{2}\right)$ and time steps $\tau_{k}=t_{k}-t_{k-1}$, $k \geq 1, t_{0}=0$. By using the implicit method for parabolic equations, we approximate the integro-parabolic differential equations in (1.1) by the difference scheme

$$
\mathcal{L}_{i} U_{i}\left(p, t_{k}\right)+f_{i}\left(p, t_{k}, U\right)+g_{i}\left(p, t_{k}, U\right)-\tau_{k}^{-1} U_{i}\left(p, t_{k-1}\right)=0,
$$

$$
\left(p, t_{k}\right) \in \omega^{h} \times \omega^{\tau}, \quad \mathcal{L}_{i} U_{i}\left(p, t_{k}\right) \equiv \mathcal{L}_{i}^{h} U_{i}\left(p, t_{k}\right)+\tau_{k}^{-1} U_{i}\left(p, t_{k}\right), i=1,2,
$$

where $\mathcal{L}_{i}^{h}, i=1,2$, are approximations of the differential operators $L_{i}, i=1,2$, from (1.1). When no confusion arises, we write $f_{i}\left(p, t_{k}, U\left(p, t_{k}\right)\right)=f_{i}\left(p, t_{k}, U\right), i=1,2$. The boundary and initial conditions are approximated by

$$
\begin{gathered}
U_{i}\left(p, t_{k}\right)=\phi_{i}\left(p, t_{k}\right), \quad\left(p, t_{k}\right) \in \partial \omega^{h} \times \omega^{\tau}, \\
U_{i}(p, 0)=\psi_{i}(p), \quad p \in \bar{\omega}^{h},
\end{gathered}
$$

where $i=1,2$, and $\partial \omega^{h}$ is the boundary of $\varpi^{h}$. The difference operators $\mathcal{L}_{i}, i=1,2$, are defined by

$$
\mathcal{L}_{i}^{h} U_{i}\left(p, t_{k}\right)=d_{i}\left(p, t_{k}\right) U_{i}\left(p, t_{k}\right)-\sum_{p^{\prime} \in \sigma_{i}^{\prime}(p)} a_{i}\left(p^{\prime}, t_{k}\right) U_{i}\left(p^{\prime}, t_{k}\right), \quad i=1,2,
$$


where $\sigma_{i}^{\prime}(p)=\sigma_{i}(p) \backslash\{p\}, \sigma_{i}(p), i=1,2$, are stencils of the scheme at an interior mesh point $p \in \omega^{h}$. We impose the following basis assumptions on the coefficients of the difference operators $\mathcal{L}_{i}^{h}, i=1,2$ :

$$
\begin{aligned}
d_{i}\left(p, t_{k}\right)>0, \quad a_{i}\left(p^{\prime}, t_{k}\right) \geq 0, \quad p^{\prime} \in \sigma_{i}^{\prime}(p), \\
d_{i}\left(p, t_{k}\right)-\sum_{p^{\prime} \in \sigma_{i}^{\prime}(p)} a_{i}\left(p^{\prime}, t_{k}\right) \geq 0, \quad\left(p, t_{k}\right) \in \omega^{h} \times \omega^{\tau} .
\end{aligned}
$$

These conditions ensure that the corresponding matrices $\mathcal{A}_{i}\left(t_{k}\right), i=$ 1,2 , are $M$-matrices, and, for any $\tau_{k}>0$, the inverse matrices $\left(I+\mathcal{A}_{i}\left(t_{k}\right)\right)^{-1}, i=1,2$, exist and are positive matrices.

We assume that the mesh $\bar{\omega}^{h}$ is connected, i.e., for two interior mesh points $\widetilde{p}$ and $\widehat{p}$, there exists a finite set of interior mesh points $\left\{p_{1}, p_{2}, \ldots, p_{s}\right\}$ such that

$$
p_{1} \in \sigma^{\prime}(\widetilde{p}), p_{2} \in \sigma^{\prime}\left(p_{1}\right), \ldots, p_{s} \in \sigma^{\prime}\left(p_{s-1}\right), \widehat{p} \in \sigma^{\prime}\left(p_{s}\right) .
$$

For linear difference problems without integral terms,

$$
\begin{aligned}
& \left(\mathcal{L}_{i}+\bar{c}_{i}\right) W_{i}\left(p, t_{k}\right)=\Phi_{i}\left(p, t_{k}\right), \quad p \in \omega^{h}, \\
& \bar{c}_{i}\left(p, t_{k}\right) \geq 0, \quad W_{i}\left(p, t_{k}\right)=\phi_{i}\left(p, t_{k}\right), \quad p \in \partial \omega^{h}, \quad i=1,2,
\end{aligned}
$$

the maximum principle and estimates to the solutions are given in the following lemma (the proof of the lemma can be found in [11]).

Lemma 2.1. Let the coefficients of the difference operators $\mathcal{L}_{i}^{h}, i=$ 1,2 , satisfy (2.2) and the mesh $\bar{\omega}^{h}$ be connected.

(i) If mesh functions $W_{i}\left(p, t_{k}\right), i=1,2$, satisfy the conditions

$$
\begin{gathered}
\left(\mathcal{L}_{i}+\bar{c}_{i}\right) W_{i}\left(p, t_{k}\right) \geq 0(\leq 0), \quad p \in \omega^{h}, \\
W_{i}\left(p, t_{k}\right) \geq 0(\leq 0), \quad p \in \partial \omega^{h},
\end{gathered}
$$

then $W_{i}\left(p, t_{k}\right) \geq 0(\leq 0), i=1,2$, in $\bar{\omega}^{h}$.

(ii) The following estimates to the solutions to (2.5) hold true:

$$
\left\|W_{i}\left(\cdot, t_{k}\right)\right\|_{\bar{\omega}^{h}} \leq \max \left\{\left\|g_{i}\left(t_{k}\right)\right\|_{\partial \omega^{h}}, \max _{p \in \omega^{h}} \frac{\left|\Phi_{i}\left(p, t_{k}\right)\right|}{\bar{c}_{i}\left(p, t_{k}\right)+\tau_{k}^{-1}}\right\}, i=1,2,
$$


where

$$
\begin{aligned}
\left\|W_{i}\left(\cdot, t_{k}\right)\right\|_{\bar{\omega}^{h}} & =\max _{p \in \bar{\omega}^{h}}\left|W_{i}\left(p, t_{k}\right)\right|, \\
\left\|g_{i}\left(\cdot, t_{k}\right)\right\|_{\partial \omega^{h}} & =\max _{p \in \partial \omega^{h}}\left|g_{i}\left(p, t_{k}\right)\right| .
\end{aligned}
$$

3. The monotone iterative method. We assume that two vector mesh functions $\widetilde{U}\left(p, t_{k}\right)=\left(\widetilde{U}_{1}\left(p, t_{k}\right), \widetilde{U}_{2}\left(p, t_{k}\right)\right)$ and $\widehat{U}\left(p, t_{k}\right)=$ $\left(\widehat{U}_{1}\left(p, t_{k}\right), \widehat{U}_{2}\left(p, t_{k}\right)\right)$ satisfy the inequalities

$$
\widetilde{U}\left(p, t_{k}\right) \geq \widehat{U}\left(p, t_{k}\right), \quad\left(p, t_{k}\right) \in \bar{\omega}^{h} \times \bar{\omega}^{\tau} .
$$

For notational convenience, we set the sector

$$
\left\langle\widehat{U}\left(t_{k}\right), \widetilde{U}\left(t_{k}\right)\right\rangle=\left\{U\left(p, t_{k}\right): \widehat{U}\left(p, t_{k}\right) \leq U\left(p, t_{k}\right) \leq \widetilde{U}\left(p, t_{k}\right), p \in \bar{\omega}^{h}\right\} .
$$

We assume that, on $\left\langle\widehat{U}\left(t_{k}\right), \widetilde{U}\left(t_{k}\right)\right\rangle$, the functions $f_{i}$ and $g_{i}^{*}, i=1,2$, satisfy the constraints

$$
\begin{array}{ll}
\frac{\partial f_{i}}{\partial u_{i}}\left(p, t_{k}, U\right) \leq c_{i}\left(p, t_{k}\right), & 0 \leq-\frac{\partial g_{i}^{*}}{\partial u_{i}}\left(p, t_{k}, t_{l}, U\right), 1 \leq l \leq k, i=1,2, \\
0 \leq-\frac{\partial f_{i}}{\partial u_{i^{\prime}}}\left(p, t_{k}, U\right), & 0 \leq-\frac{\partial g_{i}^{*}}{\partial u_{i^{\prime}}}\left(p, t_{k}, t_{l}, U\right), 1 \leq l \leq k, i \neq i^{\prime},
\end{array}
$$

where $c_{i}\left(p, t_{k}\right), i=1,2$, are nonnegative bounded functions in $\bar{\omega}^{h} \times \bar{\omega}^{\tau}$. The functions $f_{i}, i=1,2$, are said to be quasi-monotone nondecreasing if $-\partial f_{i} / \partial u_{i^{\prime}} \geq 0, i \neq i^{\prime}$, and the functions $g_{i}^{*}, i=1,2$, are said to be monotone nondecreasing if $-\partial g_{i}^{*} / \partial u_{i} \geq 0,-\partial g_{i}^{*} / \partial u_{i^{\prime}} \geq 0, i \neq i^{\prime}$.

Remark 3.1. We say that the functions $f_{i}, i=1,2$, are quasimonotone nonincreasing functions if $-\partial f_{i} / \partial u_{i^{\prime}} \leq 0, i \neq i^{\prime}$, and the functions $g_{i}^{*}, i=1,2$, are monotone nonincreasing if $-\partial g_{i}^{*} / \partial u_{i} \leq 0$, $-\partial g_{i}^{*} / \partial u_{i^{\prime}} \leq 0, i \neq i^{\prime}$. When the functions $f_{i}$ and $g_{i}^{*}, i=1,2$, are quasi-monotone nonincreasing, a transformation given by $\left(u_{1}, u_{2}\right) \rightarrow$ $\left(M-u_{1}, u_{2}\right)$ for some constant $M>0$ leads to a similar system where the functions are quasi-monotone nondecreasing.

Two vector mesh functions $\widetilde{U}\left(p, t_{k}\right)$ and $\widehat{U}\left(p, t_{k}\right)$ are called ordered upper and lower solutions of $(2.1)$, if $\widetilde{U}\left(p, t_{k}\right) \geq \widehat{U}\left(p, t_{k}\right),\left(p, t_{k}\right) \in$ 
$\bar{\omega}^{h} \times \bar{\omega}^{\tau}$, and if

$$
\begin{gathered}
\mathcal{L}_{i} \widetilde{U}_{i}\left(p, t_{k}\right)+f_{i}\left(p, t_{k}, \widetilde{U}\right)+g_{i}\left(p, t_{k}, \widetilde{U}\right)-\tau_{k}^{-1} \widetilde{U}_{i}\left(p, t_{k-1}\right) \geq 0, \\
\left(p, t_{k}\right) \in \omega^{h} \times \bar{\omega}^{\tau}, \quad \widetilde{U}_{i}\left(p, t_{k}\right) \geq \phi_{i}\left(p, t_{k}\right), \quad p \in \partial \omega^{h}, \\
\widetilde{U}_{i}(p, 0) \geq \psi_{i}(p), \quad p \in \bar{\omega}^{h}, i=1,2,
\end{gathered}
$$

and $\widehat{U}$ satisfies the above inequalities in reverse order.

A monotone iterative method for solving (2.1) is constructed in the following way. On each time level $t_{k}, k \geq 1$, we calculate $U^{(n)}\left(p, t_{k}\right)$ as follows:

$$
\begin{aligned}
\left(\mathcal{L}_{i}+c_{i}\right) Z_{i}^{(n)}\left(p, t_{k}\right)= & -\mathcal{R}_{i}\left(p, t_{k}, U^{(n-1)}\right), \quad p \in \omega^{h}, \\
\mathcal{R}_{i}\left(p, t_{k}, U^{(n-1)}\right)= & \mathcal{L}_{i} U_{i}^{(n-1)}\left(p, t_{k}\right)+f_{i}\left(p, t_{k}, U^{(n-1)}\right) \\
& +g_{i}\left(p, t_{k}, U^{(n-1)}\right)-\tau_{k}^{-1} U_{i}\left(p, t_{k-1}\right), \\
Z_{i}^{(1)}\left(p, t_{k}\right)= & \phi_{i}\left(p, t_{k}\right)-U_{i}^{(0)}\left(p, t_{k}\right), \\
Z_{i}^{(n)}\left(p, t_{k}\right)= & 0, \quad n \geq 2, p \in \partial \omega^{h}, \\
U_{i}^{(n)}\left(p, t_{k}\right)= & U_{i}^{(n-1)}\left(p, t_{k}\right)+Z_{i}^{(n)}\left(p, t_{k}\right), \quad p \in \bar{\omega}^{h}, \\
U_{i}\left(p, t_{k}\right)= & U_{i}^{\left(n\left(t_{k}\right)\right)}\left(p, t_{k}\right), \\
U_{i}(p, 0)= & \psi_{i}(p), \quad p \in \bar{\omega}^{h}, \quad i=1,2,
\end{aligned}
$$

where $\mathcal{R}_{i}\left(p, t_{k}, U^{(n-1)}\right), i=1,2$, are the residuals of the difference scheme $(2.1)$ on $U^{(n-1)}, U\left(p, t_{k}\right)$ is an approximation of the exact solution on time level $t_{k}, n\left(t_{k}\right)$ is the number of iterative steps on time level $t_{k}$ and $c_{i}\left(p, t_{k}\right), i=1,2$, are defined in (3.1).

3.1. Monotone convergence of the iterative method. We introduce the notation

$$
\begin{gathered}
F_{i}\left(p, t_{k}, U\right)=c_{i}\left(p, t_{k}\right) U_{i}\left(p, t_{k}\right)-f_{i}\left(p, t_{k}, U\right)-g_{i}\left(p, t_{k}, U\right), \\
i=1,2,
\end{gathered}
$$

and give a monotone property of $F_{i}, i=1,2$.

Lemma 3.2. Let (3.1) hold. If $U\left(p, t_{k}\right), V\left(p, t_{k}\right) \in\left\langle\widehat{U}\left(t_{k}\right), \widetilde{U}\left(t_{k}\right)\right\rangle$, such that $U\left(p, t_{k}\right) \geq V\left(p, t_{k}\right)$, then

$$
F_{i}\left(p, t_{k}, U\right) \geq F_{i}\left(p, t_{k}, V\right), \quad p \in \bar{\omega}^{h}, i=1,2 .
$$


Proof. From (3.4), we have

$$
\begin{aligned}
F_{1}\left(p, t_{k}, U\right)-F_{1}\left(p, t_{k}, V\right)= & c_{1}\left(p, t_{k}\right)\left[U_{1}\left(p, t_{k}\right)-V_{1}\left(p, t_{k}\right)\right] \\
& -\left[f_{1}\left(p, t_{k}, U_{1}, U_{2}\right)-f_{1}\left(p, t_{k}, V_{1}, U_{2}\right)\right] \\
& -\left[f_{1}\left(p, t_{k}, V_{1}, U_{2}\right)-f_{1}\left(p, t_{k}, V_{1}, V_{2}\right)\right] \\
& -\left[g_{1}\left(p, t_{k}, U_{1}, U_{2}\right)-g_{1}\left(p, t_{k}, V_{1}, U_{2}\right)\right] \\
& -\left[g_{1}\left(p, t_{k}, V_{1}, U_{2}\right)-g_{1}\left(p, t_{k}, V_{1}, V_{2}\right)\right] .
\end{aligned}
$$

By the mean-value theorem, we have

$$
\begin{gathered}
{\left[f_{1}\left(p, t_{k}, U_{1}, U_{2}\right)-f_{1}\left(p, t_{k}, V_{1}, U_{2}\right)\right]=\frac{\partial f_{1}}{\partial u_{1}}\left(t_{k}\right)\left(U_{1}\left(t_{k}\right)-V_{1}\left(p, t_{k}\right)\right),} \\
\frac{\partial f_{1}}{\partial u_{1}}\left(t_{k}\right) \equiv \frac{\partial f_{1}}{\partial u_{1}}\left(p, t_{k}, E_{1}, U_{2}\right), \quad E_{1}\left(p, t_{k}\right) \in\left\langle V_{1}\left(t_{k}\right), U_{1}\left(t_{k}\right)\right\rangle, \\
{\left[f_{1}\left(p, t_{k}, V_{1}, U_{2}\right)-f_{1}\left(p, t_{k}, V_{1}, V_{2}\right)\right]=\frac{\partial f_{1}}{\partial u_{2}}\left(t_{k}\right)\left(U_{1}\left(p, t_{k}\right)-V_{1}\left(p, t_{k}\right)\right),} \\
\frac{\partial f_{1}}{\partial u_{2}}\left(t_{k}\right) \equiv \frac{\partial f_{1}}{\partial u_{2}}\left(p, t_{k}, V_{1}, E_{2}\right), \quad E_{2}\left(p, t_{k}\right) \in\left\langle V_{2}\left(t_{k}\right), U_{2}\left(t_{k}\right)\right\rangle, \\
{\left[g_{1}\left(p, t_{k}, U_{1}, U_{2}\right)-g_{1}\left(p, t_{k}, V_{1}, U_{2}\right)\right]=\sum_{l=1}^{k} \tau_{l} \frac{\partial g_{1}^{*}}{\partial u_{1}}\left(t_{l}\right)\left(U_{1}\left(p, t_{l}\right)-V_{1}\left(p, t_{l}\right)\right),} \\
\frac{\partial g_{1}^{*}}{\partial u_{1}}\left(t_{l}\right) \equiv \frac{\partial g_{1}^{*}}{\partial u_{1}}\left(p, t_{k}, t_{l}, Q_{1}, U_{2}\right), \quad Q_{1}\left(p, t_{l}\right) \in\left\langle V_{1}\left(t_{l}\right), U_{1}\left(t_{l}\right)\right\rangle, \\
{\left[g_{1}\left(p, t_{k}, V_{1}, U_{2}\right)-g_{1}\left(p, t_{k}, V_{1}, V_{2}\right)\right]=\sum_{l=1}^{k} \tau_{l} \frac{\partial g_{1}^{*}}{\partial u_{2}}\left(t_{l}\right)\left(U_{2}\left(p, t_{l}\right)-V_{2}\left(p, t_{l}\right)\right),} \\
\frac{\partial g_{1}^{*}}{\partial u_{2}}\left(t_{l}\right) \equiv \frac{\partial g_{1}^{*}}{\partial u_{2}}\left(p, t_{k}, t_{l}, V_{1}, Q_{2}\right), \quad Q_{2}\left(p, t_{l}\right) \in\left\langle V_{2}\left(t_{l}\right), U_{2}\left(t_{l}\right)\right\rangle .
\end{gathered}
$$

From here and the assumptions of the lemma, we conclude (3.5) for $i=1$. Similarly, we can prove (3.5) for $i=2$.

Denote the sequences, generated by (3.3), by $\left\{\bar{U}^{(n)}\right\}$, if $\bar{U}^{(0)}=\widetilde{U}$ and by $\left\{\underline{U}^{(n)}\right\}$, if $\underline{U}^{(0)}=\widehat{U}$. In the following theorem, we show that these sequences converge monotonically. 
Theorem 3.3. Let the coefficients of the difference operators $\mathcal{L}_{i}^{h}$, $i=1,2$, in (2.1) satisfy (2.2), and let the computational mesh $\bar{\omega}^{h}$ be connected (2.4). Assume that $f_{i}\left(p, t_{k}, U\right)$ and $g_{i}^{*}\left(p, t_{k}, U\right), i=1,2$, satisfy (3.1), where $\widetilde{U}$ and $\widehat{U}$ are ordered upper and lower solutions (3.2) of (2.1). Then the sequences $\left\{\bar{U}^{(n)}\right\}$ and $\left\{\underline{U}^{(n)}\right\}$ are ordered upper and lower solutions and converge monotonically from, respectively, above and below, such that

$$
\underline{U}^{(n-1)}\left(p, t_{k}\right) \leq \underline{U}^{(n)}\left(p, t_{k}\right) \leq \bar{U}^{(n)}\left(p, t_{k}\right) \leq \bar{U}^{(n-1)}\left(p, t_{k}\right), \quad p \in \bar{\omega}^{h},
$$

where $k \geq 1$ and $n \geq 1$.

Proof. Since $\bar{U}^{(0)}=\widetilde{U}$ is an upper solution, then from (3.2) and (3.3) we conclude that

$$
\begin{gathered}
\left(\mathcal{L}_{i}+c_{i}\right) \bar{Z}_{i}^{(1)}\left(p, t_{1}\right) \leq 0, \quad p \in \omega^{h}, \\
\bar{Z}_{i}^{(1)}\left(p, t_{1}\right) \leq 0, \quad p \in \partial \omega^{h}, i=1,2 .
\end{gathered}
$$

From Lemma 2.1, it follows that

$$
\bar{Z}_{i}^{(1)}\left(p, t_{1}\right) \leq 0, \quad p \in \bar{\omega}^{h}, i=1,2 .
$$

Similarly, for a lower solution $\underline{U}^{(0)}=\widehat{U}$, we conclude that

$$
\underline{Z}_{i}^{(1)}\left(p, t_{1}\right) \geq 0, \quad p \in \bar{\omega}^{h}, i=1,2 .
$$

We now prove that

$$
\underline{U}_{i}^{(1)}\left(p, t_{1}\right) \leq \bar{U}_{i}^{(1)}\left(p, t_{1}\right), \quad p \in \bar{\omega}^{h}, i=1,2 .
$$

By (3.3), for $i=1,2$,

$$
\begin{aligned}
\left(\mathcal{L}_{i}+c_{i}\right) \bar{U}_{i}^{(1)}\left(p, t_{1}\right)= & c_{i}\left(p, t_{1}\right) \bar{U}_{i}^{(0)}\left(p, t_{1}\right)-f_{i}\left(p, t_{1}, \bar{U}^{(0)}\right) \\
& -g_{i}\left(p, t_{1}, \bar{U}^{(0)}\right)+\tau_{1}^{-1} U_{i}(p, 0), \quad p \in \omega^{h}, \\
\bar{U}_{i}^{(1)}\left(p, t_{1}\right)= & \phi_{i}\left(p, t_{1}\right), \quad p \in \partial \omega^{h},
\end{aligned}
$$

and

$$
\begin{aligned}
\left(\mathcal{L}_{i}+c_{i}\right) \underline{U}_{i}^{(1)}\left(p, t_{1}\right)= & c_{i} \underline{U}_{i}^{(0)}\left(p, t_{1}\right)-f_{i}\left(p, t_{1}, \underline{U}^{(0)}\right) \\
& -g_{i}\left(p, t_{1}, \underline{U}^{(0)}\right)+\tau_{1}^{-1} U_{i}(p, 0), \quad p \in \omega^{h},
\end{aligned}
$$




$$
\underline{U}_{i}^{(1)}\left(p, t_{1}\right)=\phi_{i}\left(p, t_{1}\right), \quad p \in \partial \omega^{h} .
$$

From here, taking into account that $U_{i}(p, 0)=\psi_{i}(p), i=1,2$, in the notation $W_{i}^{(n)}=\bar{U}_{i}^{(n)}-\underline{U}_{i}^{(n)}, n \geq 0, i=1,2$, we have

$$
\begin{gathered}
\left(\mathcal{L}_{i}+c_{i}\right) W_{i}^{(1)}\left(p, t_{1}\right)=F_{i}\left(p, t_{1}, \bar{U}^{(0)}\right)-F_{i}\left(p, t_{1}, \underline{U}^{(0)}\right), \quad p \in \omega^{h}, \\
W_{i}^{(1)}\left(p, t_{1}\right)=0, \quad p \in \partial \omega^{h}
\end{gathered}
$$

where $F_{i}, i=1,2$, are defined in (3.4). Since $\bar{U}^{(0)}\left(p, t_{1}\right) \geq \underline{U}^{(0)}\left(p, t_{1}\right)$, by Lemma 3.2, we conclude that the right hand sides in the difference equations are nonnegative. The positivity property in Lemma 2.1 implies $W_{i}^{(1)}\left(p, t_{1}\right) \geq 0, i=1,2$, and this leads to (3.9).

We now prove that $\bar{U}_{i}^{(1)}\left(p, t_{1}\right)$ and $\underline{U}_{i}^{(1)}\left(p, t_{1}\right), i=1,2$, are upper and lower solutions (3.2), respectively. Taking into account that

$g_{i}\left(\cdot, \bar{U}^{(n)}\right)-g_{i}\left(\cdot, \bar{U}^{(n-1)}\right)=\tau_{k}\left[g_{i}^{*}\left(\cdot, t_{k}, \bar{U}^{(n)}\right)-g_{i}^{*}\left(\cdot, t_{k}, \bar{U}^{(n-1)}\right)\right], i=1,2$,

where $g_{i}(\cdot, U)$ and $g_{i}^{*}\left(\cdot, t_{k}, U\right)$ stand, respectively, for $g_{i}\left(p, t_{k}, U\right)$ and $g_{i}^{*}\left(p, t_{k}, t_{k}, U\left(p, t_{k}\right)\right)$. From this, and by the mean-value theorem, we obtain

$g_{i}\left(\cdot, \bar{U}^{(n)}\right)-g_{i}\left(\cdot, \bar{U}^{(n-1)}\right)=\tau_{k} \frac{\partial g_{i}^{*}}{\partial u_{i}}\left(t_{k}\right) \bar{Z}_{i}^{(n)}\left(p, t_{k}\right)+\tau_{k} \frac{\partial g_{i}^{*}}{\partial u_{i^{\prime}}}\left(t_{k}\right) \bar{Z}_{i^{\prime}}^{(n)}\left(p, t_{k}\right)$,

where $i^{\prime} \neq i$, and partial derivatives are calculated at intermediate points which lie in the sector $\left\langle\bar{U}^{(n)}\left(t_{k}\right), \bar{U}^{(n-1)}\left(t_{k}\right)\right\rangle$. From this, equation (3.3), and by the mean-value theorem for $f_{i}\left(p, t_{1}, \bar{U}^{(1)}\right)$, we obtain

$$
\begin{aligned}
\mathcal{R}_{i}\left(p, t_{1}, \bar{U}^{(1)}\right)= & -\left(c_{i}-\frac{\partial f_{i}}{\partial u_{i}}\left(t_{1}\right)\right) \bar{Z}_{i}^{(1)}\left(p, t_{1}\right)+\frac{\partial f_{i}}{\partial u_{i^{\prime}}}\left(t_{1}\right) \bar{Z}_{i^{\prime}}^{(1)}\left(p, t_{1}\right) \\
& +\tau_{1} \frac{\partial g_{i}^{*}}{\partial u_{i}}\left(t_{1}\right) \bar{Z}_{i}^{(1)}\left(p, t_{1}\right)+\tau_{1} \frac{\partial g_{i}^{*}}{\partial u_{i^{\prime}}}\left(t_{1}\right) \bar{Z}_{i^{\prime}}^{(1)}\left(p, t_{1}\right),
\end{aligned}
$$

where $i^{\prime} \neq i$, and the partial derivatives are calculated at intermediate points which lie in the sector $\left\langle\bar{U}^{(1)}\left(t_{1}\right), \bar{U}^{(0)}\left(t_{1}\right)\right\rangle$. From this and equations (3.7), (3.8) and (3.9), it follows that the partial derivatives satisfy (3.1). From equations (3.1), (3.7) and (3.10), we conclude that, 
for $i=1,2$,

$$
\begin{array}{ll}
\mathcal{R}_{i}\left(p, t_{1}, \bar{U}^{(1)}\right) \geq 0, & p \in \omega^{h}, \\
\bar{U}_{i}^{(1)}\left(p, t_{1}\right)=\phi_{i}\left(p, t_{1}\right), & p \in \partial \omega^{h} .
\end{array}
$$

Thus, $\bar{U}^{(1)}\left(p, t_{1}\right)$ is an upper solution. Similarly, we can prove that $\underline{U}^{(1)}\left(p, t_{1}\right)$ is a lower solution, that is, for $i=1,2$,

$$
\begin{array}{ll}
\mathcal{R}_{i}\left(p, t_{1}, \underline{U}^{(1)}\right) \leq 0, & p \in \omega^{h}, \\
\underline{U}_{i}^{(1)}\left(p, t_{1}\right)=\phi_{i}\left(p, t_{1}\right), & p \in \partial \omega^{h} .
\end{array}
$$

By induction on $n$, we can prove that $\left\{\bar{U}^{(n)}\left(p, t_{1}\right)\right\}$ is a monotonically decreasing sequence of upper solutions and $\left\{\underline{U}^{(n)}\left(p, t_{1}\right)\right\}$ is a monotonically increasing sequence of lower solutions, which satisfy (3.6) for $t_{1}$.

From (3.6) with $t_{1}$, it follows that

$$
\widehat{U}_{i}\left(p, t_{1}\right) \leq \underline{U}_{i}^{\left(n_{1}\right)}\left(p, t_{1}\right) \leq \bar{U}_{i}^{\left(n_{1}\right)}\left(p, t_{1}\right) \leq \widetilde{U}_{i}\left(p, t_{1}\right), \quad p \in \bar{\omega}^{h}, i=1,2 .
$$

From this and by the assumption of the theorem that $\widetilde{U}\left(p, t_{2}\right)$ and $\widehat{U}\left(p, t_{2}\right)$ are, respectively, upper and lower solutions (3.2), we conclude that $\widetilde{U}\left(p, t_{2}\right)$ and $\widehat{U}\left(p, t_{2}\right)$ are upper and lower solutions with respect to $\bar{U}^{\left(n_{1}\right)}\left(p, t_{1}\right)$ and $\underline{U}^{\left(n_{1}\right)}\left(p, t_{1}\right)$, that is, for $i=1,2$,

$$
\begin{aligned}
& \mathcal{L}_{i} \widetilde{U}_{i}\left(p, t_{2}\right)+f_{i}\left(p, t_{2}, \widetilde{U}\right)+g_{i}\left(p, t_{2}, \widetilde{U}\right)-\tau_{2}^{-1} \bar{U}_{i}^{\left(n_{1}\right)}\left(p, t_{1}\right) \geq 0, \quad p \in \omega^{h}, \\
& \mathcal{L}_{i} \widehat{U}_{i}\left(p, t_{2}\right)+f_{i}\left(p, t_{2}, \widehat{U}\right)+g_{i}\left(p, t_{2}, \widehat{U}\right)-\tau_{2}^{-1} \underline{U}_{i}^{\left(n_{1}\right)}\left(p, t_{1}\right) \leq 0, \quad p \in \omega^{h} .
\end{aligned}
$$

By (3.3) with $t_{2}$, we have, for $i=1,2$,

$$
\begin{aligned}
& \left(\mathcal{L}_{i}+c_{i}\right) \bar{U}_{i}^{(1)}\left(p, t_{2}\right)=c_{i} \bar{U}_{i}^{(0)}\left(p, t_{2}\right)-f_{i}\left(p, t_{2}, \bar{U}^{(0)}\right)-g_{i}\left(p, t_{2}, \bar{U}^{(0)}\right) \\
& +\tau_{2}^{-1} \bar{U}_{i}^{\left(n_{1}\right)}\left(p, t_{1}\right), \quad p \in \omega^{h}, \quad \bar{U}_{i}^{(1)}\left(p, t_{2}\right)=\phi_{i}\left(p, t_{2}\right), \quad p \in \partial \omega^{h},
\end{aligned}
$$

and

$$
\begin{aligned}
\left(\mathcal{L}_{i}+c_{i}\right) \underline{U}_{i}^{(1)}\left(p, t_{2}\right)= & c_{i} \underline{U}_{i}^{(0)}\left(p, t_{2}\right)-f_{i}\left(p, t_{2}, \underline{U}^{(0)}\right)-g_{i}\left(p, t_{2}, \underline{U}^{(0)}\right) \\
& +\tau_{2}^{-1} \underline{U}_{i}^{\left(n_{1}\right)}\left(p, t_{1}\right), \quad p \in \omega^{h} \\
& \underline{U}_{i}^{(1)}\left(p, t_{2}\right)=\phi_{i}\left(p, t_{2}\right), \quad p \in \partial \omega^{h} .
\end{aligned}
$$


From here, we conclude that $W^{(1)}\left(p, t_{2}\right)=\bar{U}^{(1)}\left(p, t_{2}\right)-\underline{U}^{(1)}\left(p, t_{2}\right)$ satisfies

$$
\begin{aligned}
\left(\mathcal{L}_{i}+c_{i}\right) W_{i}^{(1)}\left(p, t_{2}\right)= & F_{i}\left(p, t_{2}, \bar{U}^{(0)}\right)-F_{i}\left(p, t_{2}, \underline{U}^{(0)}\right) \\
& +\tau_{2}^{-1}\left[\bar{U}_{i}^{\left(n_{1}\right)}\left(p, t_{1}\right)-\underline{U}_{i}^{\left(n_{1}\right)}\left(p, t_{1}\right)\right], \quad p \in \omega^{h}, \\
W_{i}^{(1)}\left(p, t_{2}\right)= & 0, \quad p \in \partial \omega^{h}, i=1,2 .
\end{aligned}
$$

Since $\bar{U}^{(0)}\left(p, t_{2}\right) \geq \underline{U}^{(0)}\left(p, t_{2}\right)$ and, taking into account (3.11), by Lemma 3.2 , we conclude that the right hand sides of the difference equations are nonnegative.

The positivity property in Lemma 2.1 implies $W_{i}^{(1)}\left(p, t_{2}\right) \geq 0$, $i=1,2$, and this leads to

$$
\underline{U}_{i}^{(1)}\left(p, t_{2}\right) \leq \bar{U}_{i}^{(1)}\left(p, t_{2}\right), \quad p \in \bar{\omega}^{h}, i=1,2 .
$$

The proof that $\bar{U}_{i}^{(1)}\left(p, t_{2}\right)$ and $\underline{U}_{i}^{(1)}\left(p, t_{2}\right), i=1,2$, are, respectively, upper and lower solutions, is similar to the proof of this result on the time level $t_{1}$. By induction on $n$, we can prove that $\left\{\bar{U}^{(n)}\left(p, t_{2}\right)\right\}$ is a monotonically decreasing sequence of upper solutions and $\left\{\underline{U}^{(n)}\left(p, t_{2}\right)\right\}$ is a monotonically increasing sequence of lower solutions, which satisfy (3.6) for $t_{2}$.

By induction on $k, k \geq 1$, we can prove that $\left\{\bar{U}^{(n)}\left(p, t_{k}\right)\right\}$ is a monotonically decreasing sequence of upper solutions and $\left\{\underline{U}^{(n)}\left(p, t_{k}\right)\right\}$ is a monotonically increasing sequence of lower solutions, which satisfy (3.6). Thus, the theorem is proved.

3.2. Existence and uniqueness of a solution to the nonlinear difference scheme. In this section, we investigate existence and uniqueness of a solution to the nonlinear difference scheme (2.1).

Lemma 3.4. Under the assumptions of Theorem 3.3, a solution to the nonlinear difference scheme (2.1) exists.

Proof. From (3.6), it follows that $\lim _{n \rightarrow \infty} \bar{U}^{(n)}\left(p, t_{1}\right)=\bar{V}\left(p, t_{1}\right)$, $p \in \bar{\omega}^{h}$ exists, and

$$
\bar{V}\left(p, t_{1}\right) \leq \bar{U}^{(n)}\left(p, t_{1}\right), \quad \lim _{n \rightarrow \infty} \bar{Z}^{(n)}\left(p, t_{1}\right)=0, \quad p \in \bar{\omega}^{h} .
$$


Similar to (3.10), we can prove that, for $i=1,2, n \geq 1$,

$$
\begin{aligned}
\mathcal{R}_{i}\left(p, t_{1}, \bar{U}^{(n)}\right)= & -\left(c_{i}-\frac{\partial f_{i}}{\partial u_{i}}\left(t_{1}\right)\right) \bar{Z}_{i}^{(n)}\left(p, t_{1}\right)+\frac{\partial f_{i}}{\partial u_{i^{\prime}}}\left(t_{1}\right) \bar{Z}_{i^{\prime}}^{(n)}\left(p, t_{1}\right) \\
& +\tau_{1} \frac{\partial g_{i}^{*}}{\partial u_{i}}\left(t_{1}\right) \bar{Z}_{i}^{(n)}\left(p, t_{1}\right)+\tau_{1} \frac{\partial g_{i}^{*}}{\partial u_{i^{\prime}}}\left(t_{1}\right) \bar{Z}_{i^{\prime}}^{(n)}\left(p, t_{1}\right) .
\end{aligned}
$$

From (3.14) and (3.13), we conclude that $\bar{V}\left(p, t_{1}\right)$ solves $(2.1)$ at $t_{1}$. By the assumption of Lemma 3.4 that $\widetilde{U}\left(p, t_{2}\right)$ is an upper solution and from (3.13), it follows that $\widetilde{U}\left(p, t_{2}\right)$ is an upper solution with respect to $\bar{V}\left(p, t_{1}\right)$. Using a similar argument, we can prove that the following limit

$$
\lim _{n \rightarrow \infty} \bar{U}^{(n)}\left(p, t_{2}\right)=\bar{V}\left(p, t_{2}\right), \quad p \in \bar{\omega}^{h},
$$

exists and solves (2.1) at $t_{2}$, where, according to Theorem 3.3, $\left\{\bar{U}^{(n)}\left(p, t_{2}\right)\right\}$ is a sequence of upper solutions with respect to $\bar{V}\left(p, t_{1}\right)$.

By induction on $k, k \geq 1$, we can prove that

$$
\bar{V}\left(p, t_{k}\right)=\lim _{n \rightarrow \infty} \bar{U}^{(n)}\left(p, t_{k}\right), \quad p \in \bar{\omega}^{h}, k \geq 1,
$$

is a solution of the nonlinear difference scheme (2.1). Similarly, we can prove that the mesh function $\underline{V}\left(p, t_{k}\right)$ defined by

$$
\underline{V}\left(p, t_{k}\right)=\lim _{n \rightarrow \infty} \underline{U}^{(n)}\left(p, t_{k}\right), \quad p \in \bar{\omega}^{h}, k \geq 1,
$$

is a solution of the nonlinear difference scheme (2.1).

We now impose the two-sided constraints on $f_{i}$ and $g_{i}^{*}, i=1,2$, cf., equation (3.1),

$$
\begin{aligned}
\underline{c}_{i}\left(p, t_{k}\right) & \leq \frac{\partial f_{i}}{\partial u_{i}}\left(p, t_{k}, U\right) \leq c_{i}\left(p, t_{k}\right), \\
0 & \leq-\frac{\partial f_{i}}{\partial u_{i^{\prime}}}\left(p, t_{k}, U\right) \leq \widetilde{c}_{i}\left(p, t_{k}\right),
\end{aligned}
$$




$$
\begin{gathered}
0 \leq-\frac{\partial g_{i}^{*}}{\partial u_{i}}\left(p, t_{k}, t_{l}, U\right) \leq q_{i}\left(p, t_{k}, t_{l}\right), \quad 1 \leq l \leq k, \\
0 \leq-\frac{\partial g_{i}^{*}}{\partial u_{i^{\prime}}}\left(p, t_{k}, t_{l}, U\right) \leq \widetilde{q}_{i}\left(p, t_{k}, t_{l}\right), \\
1 \leq l \leq k, \quad i \neq i^{\prime}, \quad i=1,2, \\
U\left(p, t_{k}\right) \in\left\langle\widehat{U}\left(t_{k}\right), \widetilde{U}\left(t_{k}\right)\right\rangle, \quad k \geq 1,
\end{gathered}
$$

where $\widetilde{U}, \widehat{U}$ is a pair of ordered upper and lower solutions to (2.1), $\widetilde{c}_{i}\left(p, t_{k}\right)$ and $\underline{c}_{i}\left(p, t_{k}\right), i=1,2$, are, respectively, nonnegative bounded and bounded functions in $\bar{\omega}^{h} \times \bar{\omega}^{\tau}$, and $q_{i}\left(p, t_{k}, t_{l}\right)$ and $\widetilde{q}_{i}\left(p, t_{k}, t_{l}\right)$, $i=1,2$, are positive bounded functions in $\bar{\omega}^{h} \times \bar{\omega}^{\tau}$. We also assume that time step $\tau_{k}$ satisfies the inequality

$$
\begin{gathered}
\widetilde{c}_{k}=\max _{i}\left\|\widetilde{c}_{i}\left(\cdot, t_{k}\right)\right\|_{\bar{\omega}^{h}}, \\
\underline{c}_{k}=\min _{i}\left[\min _{p \in \bar{\omega}^{h}} \underline{c}_{i}\left(p, t_{k}\right)\right], \\
\gamma_{k}=\min \left(0, \underline{c}_{k}\right), \\
\rho_{k}=\max _{i}\left\{\max _{1 \leq l \leq k}\left[\max \left(\left\|q_{i}\left(\cdot, t_{k}, t_{l}\right)\right\|_{\bar{\omega}^{h}},\left\|\widetilde{q}_{i}\left(\cdot, t_{k}, t_{l}\right)\right\|_{\bar{\omega}^{h}}\right)\right]\right\}, \quad k \geq 1 .
\end{gathered}
$$

Lemma 3.5. Let the coefficients of the difference operators $\mathcal{L}_{i}, i=$ 1,2 , in (2.1) satisfy (2.2), and let the mesh $\bar{\omega}^{h}$ be connected (2.4). Assume that $f_{i}\left(p, t_{k}, U\right)$ and $g_{i}^{*}\left(p, t_{k}, t_{l}\right), 1 \leq l \leq k, i=1,2$, satisfy, respectively, (3.15) and (3.16), where $\widetilde{U}$ and $\widehat{U}$ are ordered upper and lower solutions (3.2) of (2.1), and the mesh $\bar{\omega}^{\tau}$ satisfies (3.17). Then the nonlinear difference scheme (2.1) has a unique solution.

Proof. It suffices to show that

$$
\bar{V}\left(p, t_{k}\right)=\underline{V}\left(p, t_{k}\right), \quad p \in \bar{\omega}^{h}, k \geq 1,
$$

where $\bar{V}\left(p, t_{k}\right)$ and $\underline{V}\left(p, t_{k}\right)$ are solutions to the difference scheme (2.1), which are defined in Lemma 3.4. From equation (3.6) and Lemma 3.4, 
it follows that

$$
\begin{gathered}
\underline{U}^{(n)}\left(p, t_{k}\right) \leq \underline{V}\left(p, t_{k}\right) \leq \bar{V}\left(p, t_{k}\right) \leq \bar{U}^{(n)}\left(p, t_{k}\right), \\
p \in \bar{\omega}^{h}, \quad k \geq 1 .
\end{gathered}
$$

Letting $W\left(p, t_{k}\right)=\bar{V}\left(p, t_{k}\right)-\underline{V}\left(p, t_{k}\right)$, from (2.1), we have

$$
\begin{aligned}
\mathcal{L}_{i} W_{i}\left(p, t_{1}\right) & +\left[f_{i}\left(p, t_{1}, \bar{V}\right)-f_{i}\left(p, t_{1}, \underline{V}\right)\right] \\
& +\left[g_{i}\left(p, t_{1}, \bar{V}\right)-g_{i}\left(p, t_{1}, \underline{V}\right)\right]=0, \quad p \in \omega^{h}, \\
& W_{i}\left(p, t_{1}\right)=0, \quad p \in \partial \omega^{h}, i=1,2 .
\end{aligned}
$$

Using the mean-value theorem, we obtain

$$
\begin{gathered}
\left(\mathcal{L}_{i}+\frac{\partial f_{i}}{\partial u_{i}}\left(t_{1}\right)\right) W_{i}\left(p, t_{1}\right)=-\frac{\partial f_{i}}{\partial u_{i^{\prime}}}\left(t_{1}\right) W_{i^{\prime}}\left(p, t_{1}\right)-\tau_{1} \frac{\partial g_{i}^{*}}{\partial u_{i}}\left(t_{1}\right) W_{i}\left(p, t_{1}\right) \\
-\tau_{1} \frac{\partial g_{i}^{*}}{\partial u_{i^{\prime}}}\left(t_{1}\right) W_{i^{\prime}}\left(p, t_{1}\right), \quad p \in \omega^{h}, \\
W_{i}\left(p, t_{1}\right)=0, \quad p \in \partial \omega^{h}, \quad i^{\prime} \neq i, i=1,2,
\end{gathered}
$$

where the partial derivatives are calculated at intermediate points which lie in the sector $\left\langle\underline{V}\left(t_{1}\right), \bar{V}\left(t_{1}\right)\right\rangle$. From (3.18), it follows that the partial derivatives satisfy (3.15) and (3.16). From this, equations (2.7), (3.15) and (3.16) with $\bar{c}_{i}\left(p, t_{k}\right)=0, i=1,2$, we obtain the estimate

$$
w_{i}\left(t_{1}\right) \leq \frac{\bar{c}_{1}+2 \tau_{1} \rho_{1}}{\tau_{1}^{-1}+\left|\gamma_{1}\right|} w\left(t_{1}\right)
$$

where the following notation is in use

$$
\begin{aligned}
w_{i}\left(t_{k}\right) & =\left\|W_{i}\left(\cdot, t_{k}\right)\right\|_{\bar{\omega}^{h}}, \\
w\left(t_{k}\right) & =\max _{i} w_{i}\left(t_{k}\right), \quad k \geq 1 .
\end{aligned}
$$

By the assumption on $\tau_{1}$ in (3.17) and $w\left(t_{1}\right) \geq 0$, we conclude that $w\left(t_{1}\right)=0$. From (3.19), using the mean-value theorem, we get

$$
\begin{gathered}
\left(\mathcal{L}_{i}+\frac{\partial f_{i}}{\partial u_{i}}\left(t_{2}\right)\right) W_{i}\left(p, t_{2}\right)=-\frac{\partial f_{i}}{\partial u_{i^{\prime}}}\left(t_{2}\right) W_{i^{\prime}}\left(p, t_{2}\right)-\tau_{2} \frac{\partial g_{i}^{*}}{\partial u_{i}}\left(t_{2}\right) W_{i}\left(p, t_{2}\right) \\
--\tau_{2} \frac{\partial g_{i}^{*}}{\partial u_{i^{\prime}}}\left(t_{2}\right) W_{i^{\prime}}\left(p, t_{2}\right), \quad p \in \omega^{h}, \\
W_{i}\left(p, t_{2}\right)=0, \quad p \in \partial \omega^{h}, \quad i^{\prime} \neq i, i=1,2 .
\end{gathered}
$$


Similar to the proof that $w\left(t_{1}\right)=0$, we conclude that $w\left(t_{2}\right)=0$. Now, by induction on $k, k \geq 1$, we can prove that $w\left(t_{k}\right)=0, k \geq 1$. Thus, we have proved the lemma.

\subsection{Convergence of the monotone iterative method on each} time level. We now establish convergence properties of the iterative method (3.3) on each time level $t_{k}, k \geq 1$.

Instead of (3.15), we now assume that, in (3.15),

$$
\widetilde{c}_{k} \leq \frac{\partial f_{i}}{\partial u_{i}}\left(p, t_{k}, U\right) \leq c_{i}\left(p, t_{k}\right), \quad k \geq 1,
$$

where $\widetilde{c}_{k}$ is defined in (3.17).

Remark 3.6. We mention that the assumption $\partial f_{i} / \partial u_{i} \geq \widetilde{c}_{k}>0$ in (3.20) can always be obtained via a change of variables. Indeed, we introduce the following function $z_{i}(x, t)=e^{-\lambda t} u_{i}(x, t), i=1,2$, where $\lambda$ is a constant. Now, $z=\left(z_{1}, z_{2}\right)$ satisfies (1.1) with

$$
\begin{aligned}
\tilde{f}_{i} & =\lambda z_{i}+e^{-\lambda t} f_{i}\left(x, t, e^{\lambda t} z\right), \\
\widetilde{g}_{i}^{*} & =e^{-\lambda t} g_{i}^{*}\left(x, t, s, e^{\lambda s} z(x, s)\right),
\end{aligned}
$$

instead of $f_{i}$ and $g_{i}^{*}, i=1,2$, and we have

$$
\begin{gathered}
\frac{\partial \widetilde{f}_{i}}{\partial z_{i}}=\lambda+\frac{\partial f_{i}}{\partial u_{i}}, \quad \frac{\partial \tilde{f}_{i}}{\partial z_{i^{\prime}}}=\frac{\partial f_{i}}{\partial u_{i^{\prime}}}, \quad i^{\prime} \neq i, i=1,2 \\
-\frac{\partial \widetilde{g}_{i}^{*}}{\partial z_{i}}=-e^{-\lambda(t-s)} \frac{\partial g_{i}^{*}}{\partial u_{i}}, \quad-\frac{\partial \widetilde{g}_{i}^{*}}{\partial z_{i^{\prime}}}=-e^{-\lambda(t-s)} \frac{\partial g_{i}^{*}}{\partial u_{i^{\prime}}} \\
i^{\prime} \neq i, \quad i=1,2 .
\end{gathered}
$$

Thus, if $\lambda \geq \max _{k \geq 1}\left(\widetilde{c}_{k}+\left|\gamma_{k}\right|\right)$, where $\widetilde{c}_{k}$ and $\gamma_{k}$ are defined in (3.17), then from this and (3.15), we conclude that $\partial \widetilde{f}_{i} / \partial z_{i}$ satisfies (3.20). Since $0<e^{-\lambda(t-s)} \leq 1, \lambda>0$, then $\partial \widetilde{g}_{i}^{*} / \partial z_{i}$ and $\partial \widetilde{g}_{i}^{*} / \partial z_{i^{\prime}}$ still satisfy (3.16).

We state the discrete Gronwall's inequality from [12] in the following form. 
Lemma 3.7. Let $\left\{w_{k}\right\}$ be a sequence of nonnegative real numbers satisfying

$$
w_{k} \leq a_{k}+\sum_{l=1}^{k} b_{l} w_{l}, \quad k \geq 1
$$

where $\left\{a_{k}\right\}$ is a nondecreasing sequence of nonnegative numbers, and $b_{l} \geq 0$. Then

$$
w_{k} \leq a_{k} \exp \left(\sum_{l=1}^{k} b_{l}\right), \quad k \geq 1 \text {. }
$$

We assume that time step $\tau_{k}$ satisfies the inequality

$$
\begin{aligned}
\tau_{k} & <\sqrt{\left(\frac{\bar{c}_{k}+\widetilde{c}_{k}}{4 \rho_{k}}\right)^{2}+\frac{1}{2 \rho_{k}}}-\frac{\bar{c}_{k}+\widetilde{c}_{k}}{4 \rho_{k}}, \\
\bar{c}_{k} & =\max _{i}\left\|c_{i}\left(\cdot, t_{k}\right)\right\|_{\bar{\omega}^{h}}, \quad k \geq 1
\end{aligned}
$$

where $\widetilde{c}_{k}$ and $\rho_{k}$ are defined in (3.17), and we introduce the notation

$$
z_{i}^{(n)}\left(t_{k}\right)=\left\|Z_{i}^{(n)}\left(\cdot, t_{k}\right)\right\|_{\bar{\omega}^{h}}, \quad z^{(n)}\left(t_{k}\right)=\max _{i} z_{i}^{(n)}\left(t_{k}\right)
$$

Lemma 3.8. Let the coefficients of the difference operator $\mathcal{L}_{i}^{h},=1,2$, in (2.1) satisfy (2.2), let the mesh $\bar{\omega}^{h}$ be connected with (2.4) and the mesh $\bar{\omega}^{\tau}$ satisfy (3.21). Assume that $f_{i}\left(p, t_{k}, U\right)$ and $g_{i}^{*}\left(p, t_{k}, t_{l}, U\right)$, $i=1,2$, satisfy (3.15), (3.16) and (3.20), where $\widetilde{U}$ and $\widehat{U}$ are ordered upper and lower solutions (3.2) of (2.1). Then, for the sequences $\left\{\bar{U}^{(n)}\right\}, \bar{U}^{(0)}=\widetilde{U}$ and $\left\{\underline{U}^{(n)}\right\}, \underline{U}^{(0)}=\widehat{U}$, generated by $(3.3)$, the following estimate holds

$$
z^{(n)}\left(t_{k}\right) \leq r_{k}^{n-1} z^{(1)}\left(t_{k}\right), \quad r_{k}=\tau_{k}\left(\bar{c}_{k}+\widetilde{c}_{k}+2 \tau_{k} \rho_{k}\right)<1
$$

Proof. Using (2.7), from (3.3), we have

$$
z_{i}^{(n)}\left(t_{k}\right) \leq \tau_{k}\left\|\mathcal{R}_{i}\left(\cdot, t_{k}, U^{(n-1)}\right)\right\|_{\omega^{h}}, \quad i=1,2
$$


Similar to $(3.10)$, we can prove that

$$
\begin{aligned}
\mathcal{R}_{i}\left(p, t_{k}, U^{(n)}\right)= & -\left(c_{i}-\frac{\partial f_{i}}{\partial u_{i}}\left(t_{k}\right)\right) Z_{i}^{(n)}\left(p, t_{k}\right)+\frac{\partial f_{i}}{\partial u_{i^{\prime}}}\left(t_{k}\right) Z_{i^{\prime}}^{(n)}\left(p, t_{k}\right) \\
& +\tau_{k} \frac{\partial g_{i}^{*}}{\partial u_{i}}\left(t_{k}\right) Z_{i}^{(n)}\left(p, t_{k}\right)+\tau_{k} \frac{\partial g_{i}^{*}}{\partial u_{i^{\prime}}}\left(t_{k}\right) Z_{i^{\prime}}^{(n)}\left(p, t_{k}\right) .
\end{aligned}
$$

From equations $(3.25),(3.15),(3.16)$ and $(3.20)$, we conclude that

$$
\left\|\mathcal{R}_{i}\left(\cdot, t_{k}, U^{(n-1)}\right)\right\|_{\omega^{h}} \leq\left(\bar{c}_{k}+\widetilde{c}_{k}+2 \tau_{k} \rho_{k}\right) z^{(n-1)}\left(t_{k}\right), \quad i=1,2,
$$

where $\widetilde{c}_{k}$ and $\rho_{k}$ are defined in (3.17) and $\bar{c}_{k}$ is defined (3.21). From this and (3.24), by using (2.7), we have

$$
z^{(n)}\left(t_{k}\right) \leq \tau_{k}\left(\bar{c}_{k}+\widetilde{c}_{k}+2 \tau_{k} \rho_{k}\right) z^{(n-1)}\left(t_{k}\right)
$$

From (3.21), it follows that $r_{k}=\tau_{k}\left(\bar{c}_{k}+\widetilde{c}_{k}+2 \tau_{k} \rho_{k}\right)<1$. Thus, we have proved the lemma.

To simplify our analysis, we assume that $\tau_{k}=\tau, n\left(t_{k}\right)=n_{*}$, where $\tau$ and $n_{*}$ are constants for $k \geq 1$.

Theorem 3.9. Let all the conditions in Lemma 3.8 be satisfied. Then, for the sequences $\left\{\bar{U}^{(n)}\right\}$ and $\left\{\underline{U}^{(n)}\right\}$, the following estimate holds:

$$
\begin{gathered}
\max _{i}\left[\max _{t_{k} \in \bar{\omega}^{\tau}}\left\|U_{i}\left(\cdot, t_{k}\right)-U_{i}^{*}\left(\cdot, t_{k}\right)\right\|_{\bar{\omega}^{h}}\right] \leq C(T) r^{n-1}, \\
r=\max _{k \geq 1} r_{k}<1,
\end{gathered}
$$

where $U^{*}\left(p, t_{k}\right)$ is the unique solution to (2.1), $r_{k}$ is defined in (3.23), constant $C$ is independent of $\tau$ and the number of iterative steps on each time level $n \geq 2$.

Proof. The difference problem for $U\left(p, t_{k}\right)=U^{\left(n_{*}\right)}\left(p, t_{k}\right), k \geq 1$, can be represented in the form

$$
\begin{gathered}
\mathcal{L}_{i} U_{i}\left(p, t_{k}\right)+f_{i}\left(p, t_{k}, U\right)+g_{i}\left(p, t_{k}, U\right)-\frac{1}{\tau} U_{i}\left(p, t_{k-1}\right)=\mathcal{R}_{i}\left(p, t_{k}, U^{\left(n_{*}\right)}\right), \\
p \in \omega^{h}, \quad U_{i}\left(p, t_{k}\right)=\phi_{i}\left(p, t_{k}\right), \quad p \in \partial \omega^{h}, \quad i=1,2 .
\end{gathered}
$$


From this, equation (2.1) and using the mean-value theorem, we get the difference problem for $W_{i}\left(p, t_{k}\right)=U_{i}\left(p, t_{k}\right)-U_{i}^{*}\left(p, t_{k}\right), i=1,2$,

$$
\begin{aligned}
&\left(\mathcal{L}_{i}+\frac{\partial f_{i}}{\partial u_{i}}\left(t_{k}\right)\right) W_{i}\left(p, t_{k}\right)= \mathcal{R}_{i}\left(p, t_{k}, U\right)+\frac{1}{\tau} W_{i}\left(p, t_{k-1}\right) \\
&-\frac{\partial f_{i}}{\partial u_{i^{\prime}}}\left(t_{k}\right) W_{i^{\prime}}\left(p, t_{k}\right)-\tau \sum_{l=1}^{k} \frac{\partial g_{i}^{*}}{\partial u_{i}}\left(t_{l}\right) W_{i}\left(p, t_{l}\right) \\
&-\tau \sum_{l=1}^{k} \frac{\partial g_{i}^{*}}{\partial u_{i^{\prime}}}\left(t_{l}\right) W_{i^{\prime}}\left(p, t_{l}\right), \quad p \in \omega^{h}, \\
& W_{i}\left(p, t_{k}\right)=0, \quad p \in \partial \omega^{h}, \quad i^{\prime} \neq i, \quad i=1,2,
\end{aligned}
$$

where the partial derivatives are calculated at intermediate points, which lie between $U\left(p, t_{k}\right)$ and $U^{*}\left(p, t_{k}\right)$. Thus, the partial derivatives satisfy equations (3.16) and (3.20). From equations (3.27) and (3.25), for $k \geq 1, p \in \omega^{h}$, we conclude that

$$
\begin{aligned}
\left(\mathcal{L}_{i}+\frac{\partial f_{i}}{\partial u_{i}}\left(t_{k}\right)\right) W_{i}\left(p, t_{k}\right)= & -\left(c_{i}-\frac{\partial f_{i}}{\partial u_{i}}\left(t_{k}\right)\right) Z_{i}\left(p, t_{k}\right) \\
& +\frac{\partial f_{i}}{\partial u_{i^{\prime}}}\left(t_{k}\right) Z_{i^{\prime}}\left(p, t_{k}\right)+\tau \frac{\partial g_{i}^{*}}{\partial u_{i}}\left(t_{k}\right) Z_{i}\left(p, t_{k}\right) \\
& +\tau \frac{\partial g_{i}^{*}}{\partial u_{i^{\prime}}}\left(t_{k}\right) Z_{i^{\prime}}\left(p, t_{k}\right)+\frac{1}{\tau} W_{i}\left(p, t_{k-1}\right) \\
& -\frac{\partial f_{i}}{\partial u_{i^{\prime}}}\left(t_{k}\right) W_{i^{\prime}}\left(p, t_{k}\right)-\sum_{l=1}^{k} \tau \frac{\partial g_{i}^{*}}{\partial u_{i}}\left(t_{l}\right) W_{i}\left(p, t_{l}\right) \\
& -\sum_{l=1}^{k} \tau \frac{\partial g_{i}^{*}}{\partial u_{i^{\prime}}}\left(t_{l}\right) W_{i^{\prime}}\left(p, t_{l}\right), \quad i^{\prime} \neq i,
\end{aligned}
$$

where $Z_{i}\left(p, t_{k}\right)=Z_{i}^{\left(n_{*}\right)}\left(p, t_{k}\right), i=1,2$. From equations (3.28), (3.15), (3.16) and (3.20), by using (2.7), and in the notation of (3.19) and (3.22), we have

$$
\begin{aligned}
w\left(t_{k}\right) \leq \frac{1}{\tau^{-1}+\widetilde{c}_{k}}\left[\left(\bar{c}_{k}+\widetilde{c}_{k}+2 \tau \rho_{k}\right)\right. & z\left(t_{k}\right)+\widetilde{c}_{k} w\left(t_{k}\right) \\
& \left.+\sum_{l=1}^{k} 2 \tau \rho_{l} w\left(t_{l}\right)+\tau^{-1} w\left(t_{k-1}\right)\right],
\end{aligned}
$$


where $\widetilde{c}_{k}, \underline{c}_{k}, \rho_{k}$ and $\bar{c}_{k}$ are defined in (3.17) and (3.21), respectively. From this and equation (3.23), we obtain the estimate (3.29)

$$
w\left(t_{k}\right) \leq \tau\left(\bar{c}_{k}+\widetilde{c}_{k}+2 \tau \rho_{k}\right) r_{k}^{n-1} z^{(1)}\left(t_{k}\right)+\sum_{l=1}^{k} 2 \tau^{2} \rho_{l} w\left(t_{l}\right)+w\left(t_{k-1}\right) .
$$

From (3.3) and by (2.7),

$$
\begin{aligned}
z_{i}^{(1)}\left(t_{1}\right) \leq & \tau \| \mathcal{L}_{i} U_{i}^{(0)}\left(\cdot, t_{1}\right)+f_{i}\left(\cdot, t_{1}, U^{(0)}\right) \\
& +g_{i}\left(\cdot, t_{1}, U^{(0)}\right)-\tau^{-1} U_{i}\left(\cdot, t_{0}\right) \|_{\omega^{h}} .
\end{aligned}
$$

Since $U^{(0)}\left(p, t_{1}\right)$ and $U\left(p, t_{0}\right)$ are independent of $\tau$, for sufficiently small $\tau, z_{i}^{(1)}\left(t_{1}\right), i=1,2$, are independent of $\tau$, that is,

$$
z^{(1)}\left(t_{1}\right) \leq A_{1}
$$

where the constant $A_{1}$ is independent of $\tau$. Now by induction on $k$, we prove that

$$
z^{(1)}\left(t_{k}\right) \leq A_{k}, \quad k \geq 1
$$

where constants $A_{k}, k \geq 1$, are independent of $\tau$. From equations (3.30) and (3.29), we have

$$
w\left(t_{k}\right) \leq B \tau r^{n-1}+2 \tau^{2} \rho \sum_{l=1}^{k} w\left(t_{l}\right)+w\left(t_{k-1}\right),
$$

where the constant $B$ is independent of $\tau$, such that

$$
B \geq \max _{k \geq 1}\left[\left(\bar{c}_{k}+\widetilde{c}_{k}+2 T \rho_{k}\right) A_{k}\right], \quad \rho=\max _{k \geq 1} \rho_{k} .
$$

From this and taking into account that $w\left(t_{0}\right)=0$, by induction on $k$, we prove the inequality

$$
w\left(t_{k}\right) \leq B(k \tau) r^{n-1}+2 \tau^{2} \rho\left(\sum_{l=1}^{k}(k-l+1) w\left(t_{l}\right)\right) .
$$

By Lemma 3.7 with $a_{k}=B(k \tau) r^{n-1}, k \geq 1$ and $b_{l}=2 \tau^{2} \rho(k-l+1)$, $1 \leq l \leq k$, we get

$$
w\left(t_{k}\right) \leq B(k \tau) r^{n-1} \exp \left(2 \tau^{2} \rho \sum_{l=1}^{k} l\right) .
$$


From this and taking into account that

$$
\sum_{l=1}^{k} l \leq k^{2} / 2, \quad k \tau \leq T,
$$

we prove equation (3.26) with $C(T)=B T \exp \left(\rho T^{2}\right)$.

Remark 3.10. The implicit two-level difference scheme (2.1) is of first order with respect to time steps. As follows from $(3.23)$, if $\bar{c}_{k}=\mathcal{O}(1)$, $\widetilde{c}_{k}=\mathcal{O}(1)$ and $\rho_{k}=\mathcal{O}(1), k \geq 1$, then $r_{k}=\mathcal{O}(\tau)$. To guarantee the consistency of the global errors in the implicit difference scheme and in the monotone iterative method (3.3) we can choose $n=2$ in (3.26).

3.4. Convergence of the monotone iterative method to the solution of the nonlinear difference scheme on $[0, T]$. We now investigate convergence of the monotone iterative method (3.3) to the solution of the nonlinear difference scheme (2.1). We choose the stopping criterion of the iterative method (3.3) in the form

$$
\max _{i}\left\|\mathcal{R}_{i}\left(\cdot, t_{k}, U^{(n)}\right)\right\|_{\omega^{h}} \leq \delta,
$$

where $\delta$ is a prescribed accuracy and set up $U\left(p, t_{k}\right)=U^{\left(n_{k}\right)}\left(p, t_{k}\right)$, $p \in \bar{\omega}^{h}$, such that $n\left(t_{k}\right)$ is minimal subject to $(3.32)$.

We assume that time step $\tau_{k}$ satisfies the inequality

$$
\tau_{k}<\sqrt{\left(\frac{\widetilde{c}_{k}}{4 \rho_{k}}\right)^{2}+\frac{1}{2 \rho_{k}}}-\frac{\widetilde{c}_{k}}{2 \rho_{k}}, \quad k \geq 1 .
$$

To simplify our analysis, we assume that $\tau_{k}=\tau, k \geq 1$, and with the aid of Lemma 3.7, prove the following convergence result for the iterative method (3.3), (3.32).

Theorem 3.11. Assume that the mesh $\bar{\omega}^{\tau}$ satisfies (3.33), and let all other conditions in Lemma 3.8 be satisfied. Then, for the sequences $\left\{\bar{U}^{(n)}\right\}$ and $\left\{\underline{U}^{(n)}\right\}$ generated by equations (3.3) and (3.32), the following estimate holds:

$$
\max _{i}\left[\max _{t_{k} \in \bar{\omega}^{\tau}}\left\|U_{i}\left(\cdot, t_{k}\right)-U_{i}^{*}\left(\cdot, t_{k}\right)\right\|_{\bar{\omega}^{h}}\right] \leq C(T) \delta
$$


where $U^{*}\left(p, t_{k}\right)$ is the unique solution to (2.1), and the constant $C$ is independent of $\tau$.

Proof. The existence and uniqueness of the solution to (2.1) have been proved in Lemmas 3.4 and 3.5, respectively. From equations (3.16), (3.20) and (3.27), using equation (2.7) and taking into account that, according to Theorem 3.3 the stopping criterion (3.32) can always be satisfied, we have

$$
\begin{gathered}
w_{i}\left(t_{k}\right) \leq \frac{1}{\widetilde{c}_{k}+\tau^{-1}}\left[\delta+\tau^{-1} w_{i}\left(t_{k-1}\right)+\widetilde{c}_{k} w_{i^{\prime}}\left(t_{k}\right)+\tau \rho \sum_{l=1}^{k}\left(w_{i}\left(t_{l}\right)+w_{i^{\prime}}\left(t_{l}\right)\right)\right], \\
\rho=\max _{k \geq 1} \rho_{k}, \quad w_{i}\left(t_{k}\right)=\left\|W_{i}\left(\cdot, t_{k}\right)\right\|_{\bar{\omega}^{h}}, \quad i^{\prime} \neq i, \quad i=1,2,
\end{gathered}
$$

where $\rho_{k}$ is defined in equation (3.17). From this, in the notation $w\left(t_{k}\right)=\max _{i} w_{i}\left(t_{k}\right)$, we have

$$
w\left(t_{k}\right) \leq \frac{1}{\widetilde{c}_{k}+\tau^{-1}}\left[\delta+\tau^{-1} w\left(t_{k-1}\right)+\widetilde{c}_{k} w\left(t_{k}\right)+2 \tau \rho \sum_{l=1}^{k} w\left(t_{l}\right)\right] .
$$

Thus, taking into account that

$$
1-\frac{\widetilde{c}_{k}}{\widetilde{c}_{k}+\tau^{-1}}>0
$$

we obtain the inequality

$$
w\left(t_{k}\right) \leq w\left(t_{k-1}\right)+2 \tau^{2} \rho \sum_{l=1}^{k} w\left(t_{l}\right)+\tau \delta .
$$

From this and taking into account that $w\left(t_{0}\right)=0$, by induction on $k$, we prove the following inequality:

$$
w\left(t_{k}\right) \leq k \tau \delta+2 \tau^{2} \rho \sum_{l=1}^{k}(k-l+1) w\left(t_{l}\right) .
$$

By Lemma 3.7 with $a_{k}=k \tau \delta, k \geq 1$ and $b_{l}=2 \tau^{2} \rho(k-l+1), 1 \leq l \leq k$, we get

$$
w\left(t_{k}\right) \leq(k \tau \delta) \exp \left(2 \tau^{2} \rho \sum_{l=1}^{k} l\right)
$$


From here and taking into account that $\sum_{l=1}^{k} l \leq k^{2} / 2, k \tau \leq T$, we prove equation (3.34) with $C(T)=T \exp \left(\rho T^{2}\right)$.

3.5. Convergence of the nonlinear difference scheme (2.1) to the solution of problem (1.1). The following notation:

$$
\begin{aligned}
E\left(p, t_{k}\right) & =U^{*}\left(p, t_{k}\right)-u^{*}\left(p, t_{k}\right), & e_{i}\left(t_{k}\right) & =\left\|E_{i}\left(\cdot, t_{k}\right)\right\|_{\bar{\omega}^{h}}, \\
e\left(t_{k}\right) & =\max _{i} e_{i}\left(t_{k}\right), & \xi_{i}\left(t_{k}\right) & =\left\|\Xi_{i}\left(\cdot, t_{k}\right)\right\|_{\omega^{h}}, \\
\xi\left(t_{k}\right) & =\max _{i} \xi_{i}\left(t_{k}\right), & \xi & =\max _{k \geq 1} \xi\left(t_{k}\right),
\end{aligned}
$$

is introduced, where $U^{*}\left(p, t_{k}\right)$ and $u^{*}\left(p, t_{k}\right)$ are unique solutions to, respectively, equations (2.1) and (1.1), and $\Xi\left(p, t_{k}\right)$ is the local truncation error of $u^{*}(x, t)$ on the nonlinear difference scheme (2.1). To simplify our analysis, we assume that $\tau_{k}=\tau, k \geq 1$.

The following theorem presents convergence of the nonlinear difference scheme (2.1) to the solution of problem (1.1).

Theorem 3.12. Let all the conditions in Theorem 3.11 be satisfied. Then the error in the nonlinear difference scheme (2.1) satisfies the inequality

$$
e\left(t_{k}\right) \leq C(T) \xi,
$$

where the constant $C$ is independent of $\tau$.

Proof. Under the assumptions of Theorem 3.11 on $f_{i}$ and $g_{i}^{*}, i=1,2$, the nonlinear integro-parabolic problem (1.1) has a unique solution (see [9, page 73, Theorem 6.1], for details). From (2.1), by the mean-value theorem, we get the difference problem for the error $E\left(p, t_{k}\right)$

$$
\begin{aligned}
&\left(\mathcal{L}_{i}+\frac{\partial f_{i}}{\partial u_{i}}\left(t_{k}\right)\right) E_{i}\left(p, t_{k}\right)=-\frac{\partial f_{i}}{\partial u_{i^{\prime}}} E_{i^{\prime}}\left(p, t_{k}\right)-\sum_{l=1}^{k} \tau \frac{\partial g_{i}^{*}}{\partial u_{i}}\left(t_{l}\right) E_{i}\left(p, t_{l}\right) \\
&-\sum_{l=1}^{k} \tau \frac{\partial g_{i}^{*}}{\partial u_{i^{\prime}}}\left(t_{l}\right) E_{i^{\prime}}\left(p, t_{l}\right)+\frac{1}{\tau} E_{i}\left(p, t_{k-1}\right) \\
&-\Xi_{i}\left(p, t_{k}\right), \\
& p \in \omega^{h}, \quad E_{i}\left(p, t_{k}\right)=0, \quad p \in \partial \omega^{h}, \quad E_{i}(p, 0)=0, \quad p \in \bar{\omega}^{h},
\end{aligned}
$$


where the partial derivatives are calculated at intermediate points, which lie between $U\left(p, t_{k}\right)$ and $u\left(p, t_{k}\right)$. From this and equations (3.16) and (3.20), and by using (2.7), we have

$$
\begin{gathered}
e_{i}\left(t_{k}\right) \leq \frac{1}{\widetilde{c}_{k}+\tau^{-1}}\left[\widetilde{c}_{k} e_{i^{\prime}}\left(t_{k}\right)+\tau \rho \sum_{l=1}^{k}\left(e_{i}\left(t_{l}\right)+e_{i^{\prime}}\left(t_{l}\right)\right)\right. \\
\left.+\frac{1}{\tau} e_{i}\left(t_{k-1}\right)+\xi_{i}\left(t_{k}\right)\right]
\end{gathered}
$$

where $\rho=\max _{k \geq 1} \rho_{k}$ and $\rho_{k}, k \geq 1$, are defined in (3.17). From this, we obtain

$$
e\left(t_{k}\right) \leq \frac{1}{\widetilde{c}_{k}+\tau^{-1}}\left[\widetilde{c}_{k} e\left(t_{k}\right)+2 \tau \rho \sum_{l=1}^{k} e\left(t_{l}\right)+\frac{1}{\tau} e\left(t_{k-1}\right)+\xi\right] .
$$

Thus, taking into account that

$$
1-\frac{\widetilde{c}_{k}}{\widetilde{c}_{k}+\tau^{-1}}>0
$$

we obtain the inequality

$$
e\left(t_{k}\right) \leq e\left(t_{k-1}\right)+2 \tau^{2} \rho \sum_{l=1}^{k} e\left(t_{l}\right)+\tau \xi
$$

From this and $e\left(t_{0}\right)=0$, by induction on $k$, we prove the following inequality:

$$
e\left(t_{k}\right) \leq k \tau \xi+2 \tau^{2} \rho \sum_{l=1}^{k}(k-l+1) e\left(t_{l}\right) .
$$

By Lemma 3.7 with $a_{k}=k \tau \xi, k \geq 1$ and $b_{l}=2 \tau^{2} \rho(k-l+1), 1 \leq l \leq k$, we get

$$
e\left(t_{k}\right) \leq(k \tau \xi) \exp \left(2 \tau^{2} \rho \sum_{l=1}^{k} l\right)
$$

Taking into account that $\sum_{l=1}^{k} l \leq k^{2} / 2, k \tau \leq T$, we prove (3.35) with $C(T)=T \exp \left(\rho T^{2}\right)$.

We now formulate the discrete problem (2.1) obtained by the finite difference method. Consider the rectangular mesh $\bar{\omega}^{h}$ of the form

$$
\bar{\omega}^{h}=\left\{x_{j_{\alpha}}=j_{\alpha} h_{\alpha}, j_{\alpha}=0,1, \ldots, M_{\alpha} ; \alpha=1, \ldots, \kappa\right\} .
$$


Let $j=\left(j_{1}, \ldots, j_{\kappa}\right)$ be a multiple index with $j_{\alpha}=0,1, \ldots, M_{\alpha}$, and let $x_{j}=\left(x_{j_{1}}, \ldots, x_{j_{\kappa}}\right)$ be an arbitrary mesh point in $\bar{\omega}^{h}$. We use the standard central difference approximations for the first and second order spatial partial derivatives

$$
\begin{aligned}
\delta_{i}^{(\alpha)} U_{i}\left(x_{j}, t_{k}\right) & =\frac{1}{2 h_{\alpha}}\left[U_{i}\left(x_{j}^{\left(+1_{\alpha}\right)}, t_{k}\right)-U_{i}\left(x_{j}^{\left(-1_{\alpha}\right)}, t_{k}\right)\right], \\
\Delta_{i}^{(\alpha)} U_{i}\left(x_{j}, t_{k}\right) & =\frac{1}{h_{\alpha}^{2}}\left[U_{i}\left(x_{j}^{\left(+1_{\alpha}\right)}, t_{k}\right)-2 U_{i}\left(x_{j}, t_{k}\right)+U_{i}\left(x_{j}^{\left(-1_{\alpha}\right)}, t_{k}\right)\right], \\
x_{j}^{\left( \pm 1_{\alpha}\right)} & =\left(x_{j_{1}}, \ldots, x_{j_{\alpha}-1}, x_{j_{\alpha}} \pm h_{\alpha}, x_{j_{\alpha}+1}, \ldots, x_{j_{M_{\alpha}}}\right),
\end{aligned}
$$

Thus, the difference operators $\mathcal{L}_{i}^{h}, i=1,2$, in (2.1) are defined by

$$
\mathcal{L}_{i}^{h} U_{i}\left(x_{j}, t_{k}\right)=\sum_{\alpha=1}^{\kappa}\left[\left(D_{i}\left(x_{j}, t_{k}\right) \Delta_{i}^{(\alpha)}+v_{i, \alpha}\left(x_{j}, t_{k}\right) \delta_{i}^{(\alpha)}\right) U\left(x_{j}, t_{k}\right)\right] .
$$

To ensure that the conditions in (2.2) are satisfied, we choose

$$
h_{\alpha} \leq \min _{i, j, k} \frac{2 D_{i}\left(x_{j}, t_{k}\right)}{\left|v_{i, \alpha}\left(x_{j}, t_{k}\right)\right|}, \quad \alpha=1, \ldots, \kappa .
$$

For this finite difference scheme, the maximum of the local truncation error $\xi$ in Theorem 3.12 is estimated as $\xi=\mathcal{O}\left(\tau+h^{2}\right)$, where $h=$ $\max _{\alpha} h_{\alpha}$. If the effect of convection dominates diffusion to the extent that condition (3.36) requires a prohibitively small $h_{\alpha}$, then an upwind scheme can be used to remove any restrictions on $h_{\alpha}$. In this case, the local truncation error $\xi$ is estimated as $\xi=\mathcal{O}(\tau+h)$.

4. Construction of initial upper and lower solutions. Here, we give some conditions on functions $f_{i}$ and $g_{i}^{*}, i=1,2$, to guarantee the existence of upper $\widetilde{U}$ and lower $\widehat{U}$ solutions, which are used as the initial iterations in the monotone iterative method (3.3).

4.1. Bounded functions. Let functions $f_{i}, g_{i}^{*}, \phi_{i}$ and $\psi_{i}, i=1,2$, from (1.1) satisfy the following conditions:

$$
\begin{aligned}
& f_{i}(x, t, 0) \leq 0, \quad g_{i}^{*}(x, t, s, 0) \leq 0, \quad \phi_{i}(x, t) \geq 0, \quad \psi_{i}(x) \geq 0, \\
& f_{i}(x, t, u) \geq-\chi_{i}, \quad g_{i}^{*}(x, t, s, u) \geq-\nu_{i}, \quad u_{i} \geq 0, \quad i=1,2,
\end{aligned}
$$

where $\chi_{i}$ and $\nu_{i}, i=1,2$, are positive constants. 
From (4.1) and (3.2), it follows that the functions

$$
\widehat{U}_{i}\left(p, t_{k}\right)=\left\{\begin{array}{ll}
\psi_{i}(p), & k=0, \\
0, & k \geq 1,
\end{array} \quad p \in \bar{\omega}^{h}, \quad i=1,2,\right.
$$

are lower solutions of (2.1).

We introduce the linear problems for $i=1,2$,

$$
\begin{aligned}
\mathcal{L}_{i}\left(p, t_{k}\right) \widetilde{U}_{i}\left(p, t_{k}\right) & =\tau_{k}^{-1} \widetilde{U}_{i}\left(p, t_{k-1}\right)+\chi_{i}+\nu_{i} t_{k}, & & p \in \omega^{h}, \quad k \geq 1, \\
\widetilde{U}_{i}\left(p, t_{k}\right) & =\phi_{i}\left(p, t_{k}\right), & & p \in \partial \omega^{h}, k \geq 1, \\
\widetilde{U}_{i}(p, 0) & =\psi_{i}(p), & & p \in \bar{\omega}^{h} .
\end{aligned}
$$

Lemma 4.1. Let the conditions in (4.1) be satisfied. Then $\widehat{U}$ and $\widetilde{U}$ from, respectively, (4.2) and (4.3), are ordered lower and upper solutions to $(2.1)$, such that

$$
0 \leq \widehat{U}_{i}\left(p, t_{k}\right) \leq \widetilde{U}_{i}\left(p, t_{k}\right), \quad p \in \bar{\omega}^{h}, k \geq 0, i=1,2 .
$$

Proof. From (4.1) and (4.3), by the maximum principle in Lemma 2.1, we conclude (4.4), for $k=1$,

$$
\widetilde{U}_{i}\left(p, t_{1}\right) \geq 0, \quad p \in \bar{\omega}^{h}, i=1,2 .
$$

By induction on $k$, we prove (4.4) for $k \geq 1$. We now show that $\widetilde{U}$ is an upper solution (3.2) to (2.1). From (3.2), (3.3), (4.1) and (4.3), we have for $i=1,2$,

$$
\begin{aligned}
\mathcal{R}_{i}\left(p, t_{k}, \widetilde{U}\right)= & \mathcal{L}_{i}\left(p, t_{k}\right) \widetilde{U}_{i}\left(p, t_{k}\right)+f_{i}\left(p, t_{k}, \widetilde{U}\right) \\
& +g_{i}\left(p, t_{k}, \widetilde{U}\right)-\tau_{k}^{-1} \widetilde{U}_{i}\left(p, t_{k-1}\right) \\
= & {\left[\chi_{i}+f_{i}\left(p, t_{k}, \widetilde{U}\right)\right]+\left[\nu_{i} t_{k}+g_{i}\left(p, t_{k}, \widetilde{U}\right)\right] \geq 0, }
\end{aligned}
$$

where $p \in \omega^{h}$. Since $\widetilde{U}$ satisfies the boundary-initial conditions, we prove that $\widetilde{U}$ is an upper solution to (2.1). From this and (4.4), we conclude that $\widehat{U}$ and $\widetilde{U}$ from, respectively, (4.2) and (4.3), are ordered lower and upper solutions to (2.1). 
4.2. Constant upper and lower solutions. Let the functions $f_{i}$, $g_{i}^{*}, \phi_{i}$ and $\psi_{i}, i=1,2$, from (1.1) satisfy the following conditions.

$$
\begin{array}{rlrl}
f_{i}(x, t, 0) & \leq 0, & & g_{i}^{*}(x, t, s, 0) \leq 0, \\
\phi_{i}(x, t) & \geq 0, \quad & \psi_{i}(x) \geq 0, \quad i=1,2 .
\end{array}
$$

It is clear that the functions from (4.2) are lower solutions of (2.1).

We assume that there exist positive constants $M_{i}, i=1,2$, such that

$$
\begin{gathered}
f_{i}\left(p, t_{k}, M\right)+\sum_{l=1}^{k} \tau_{l} g_{i}^{*}\left(p, t_{k}, t_{l}, M\right) \geq 0, \quad M=\left(M_{1}, M_{2}\right), \\
\phi_{i}\left(p, t_{k}\right) \leq M_{i}, \quad p \in \partial \omega^{h}, \\
\psi_{i}(p) \leq M_{i}, \quad p \in \bar{\omega}^{h}, \quad i=1,2, \quad k \geq 1,
\end{gathered}
$$

and introduce the functions

$$
\widetilde{U}_{i}\left(p, t_{k}\right)=\left\{\begin{array}{ll}
\psi_{i}(p), & k=0, \\
M_{i}, & k \geq 1,
\end{array} \quad p \in \bar{\omega}^{h}, i=1,2 .\right.
$$

Lemma 4.2. Let conditions (4.5) and (4.6) be satisfied. Then $\widehat{U}$ and $\widetilde{U}$ from, respectively, (4.2) and (4.8), are ordered lower and upper solutions to (2.1) and satisfy (4.4).

Proof. The proof of Lemma 4.2 repeats the proof of Lemma 4.1, with the following modification:

$$
\begin{aligned}
\mathcal{R}_{i}\left(p, t_{k}, \widetilde{U}\right)= & \mathcal{L}_{i}\left(p, t_{k}\right) \widetilde{U}_{i}\left(p, t_{k}\right)+f_{i}\left(p, t_{k}, \widetilde{U}\right)+g_{i}\left(p, t_{k}, \widetilde{U}\right) \\
& -\tau_{k}^{-1} \widetilde{U}_{i}\left(p, t_{k-1}\right) \geq f_{i}\left(p, t_{k}, M\right)+g_{i}\left(p, t_{k}, M\right) \geq 0
\end{aligned}
$$

where $p \in \omega^{h}$.

5. Numerical experiments. In this section, we give applications of the monotone iterative method (3.3) for numerical solutions of three test problems. Exact solutions of the test problems are unknown, and numerical solutions, obtained by the monotone iterative method, are compared to reference solutions.

We choose the stopping criterion in the form (3.32) with $\delta=10^{-5}$. In all of the numerical experiments, the monotone property of upper 
and lower solutions is observed at each mesh point of the computational domain.

Example 5.1. In

$$
\omega=\left\{0<x_{1}<1,0<x_{2}<1\right\},
$$

we consider the following test problem:

$$
\begin{gathered}
\frac{\partial u_{1}}{\partial t}-D_{1} \Delta u_{1}+\zeta_{1} u_{1}\left(1+e^{-u_{2}}\right)-\int_{0}^{t} u_{2}(x, s) d s=0, \\
\frac{\partial u_{2}}{\partial t}-D_{2} \Delta u_{2}+\zeta_{2} u_{2}\left(1+\frac{1}{1+u_{1}}\right)-\int_{0}^{t} u_{1}(x, s) d s=0, \\
(x, t) \in \omega \times(0, T], \quad u_{i}(x, t)=0, \quad(x, t) \in \partial \omega \times(0, T], \\
u_{i}(x, 0)=\sin \left(\pi x_{1}\right) \sin \left(\pi x_{2}\right), \quad x \in \bar{\omega},
\end{gathered}
$$

where $\Delta u=\left(u_{x_{1} x_{1}}+u_{x_{2} x_{2}}\right)$, and $D_{i}, \zeta_{i}, i=1,2$, are positive constants. For this test problem, we have

$$
f_{1}=\zeta_{1} u_{1}\left(1+e^{-u_{2}}\right), \quad f_{2}=\zeta_{2} u_{2}\left(1+\frac{1}{1+u_{1}}\right), \quad g_{i}^{*}=-u_{i^{\prime}}, i \neq i^{\prime},
$$

where, for $u_{i} \geq 0, i=1,2$,

$$
\begin{aligned}
0 \leq \frac{\partial f_{1}}{\partial u_{1}}=\zeta_{1}\left(1+e^{-u_{2}}\right) \leq 2 \zeta_{1}, & 0 \leq-\frac{\partial f_{1}}{\partial u_{2}}=\zeta_{1} u_{1} e^{-u_{2}}, \\
0 \leq \frac{\partial f_{2}}{\partial u_{2}}=\zeta_{2}\left(1+\frac{1}{1+u_{1}}\right) \leq 2 \zeta_{2}, & 0 \leq-\frac{\partial f_{2}}{\partial u_{1}}=\frac{\zeta_{2} u_{2}}{\left(1+u_{1}\right)^{2}} \\
\frac{\partial g_{i}^{*}}{\partial u_{i}}=0, \quad-\frac{\partial g_{i}^{*}}{\partial u_{i^{\prime}}}=1, & i \neq i^{\prime}, i=1,2 .
\end{aligned}
$$

From this, we choose $c_{i}=2 \zeta_{i}, i=1,2$, in the monotone iterative method (3.3).

The conditions in (4.5) hold true without any extra restrictions.

To guarantee (4.6), we assume that $M_{i}, i=1,2$, satisfies the inequalities

$$
\zeta_{1} M_{1}-M_{2} T \geq 0, \quad \zeta_{2} M_{2}-M_{1} T \geq 0 .
$$

Thus, if we choose $M_{1}=M_{2}=M \geq 1$ and denote $\zeta=\min \left(\zeta_{1}, \zeta_{2}\right)$, then impose the condition $\zeta \geq T$, by Lemma 4.2 , we conclude that $\widehat{U}$ 
and $\widetilde{U}$ from, respectively, (4.2) and (4.8), are ordered lower and upper solutions and satisfy (4.4).

We discretize the differential problem by the finite difference approximation on an uniform space mesh with the step size $h_{1}=h_{2}=h$ $(N=1 / h)$.

In Table 1, for the two sets of parameters $D_{i}=1, \zeta_{i}=2, i=1,2$, and $D_{i}=0.1, \zeta_{i}=10, i=1,2$, we present the numerical error

$$
\operatorname{error}(h)=\max _{i=1,2}\left[\left\|\widehat{U}_{i}(\cdot, T)-\widehat{U}_{i}^{\mathrm{ref}}(\cdot, T)\right\|_{\bar{\omega}^{h}}\right], \quad T=1,
$$

where $\widehat{U}_{i}^{\text {ref }}\left(p, t_{k}\right), i=1,2$, are reference solutions with $N=128$, the order of the numerical error

$$
\operatorname{order}(h)=\log _{2}\left(\frac{\operatorname{error}(h)}{\operatorname{error}(h / 2)}\right),
$$

and numbers of monotone iterations on each time level for different mesh sizes $h$ and $\tau=h^{2}$. The data in the table indicate that the numerical solution has the second-order accuracy in the space variables, and numbers of iterations decrease as $N$ increases. We mention here that numerical experiments show that if, in the reference solution, $N$ increases, then the order of the numerical error tends to the second one.

In Table 2, for the same two sets of parameters as in Table 1, we present the numerical error based on lower solutions as in Table 1, the order of the numerical error and numbers of monotone iterations on

TABLE 1. Numerical results for Example 5.1 with $\tau=h^{2}$.

\begin{tabular}{cccccc}
\hline$N$ & 4 & 8 & 16 & 32 & 64 \\
\hline \multicolumn{5}{c}{$D_{i}=1, \zeta_{i}=2, i=1,2$} \\
\hline error & $5.120 \mathrm{e}-4$ & $1.276 \mathrm{e}-4$ & $3.157 \mathrm{e}-5$ & $7.533 \mathrm{e}-6$ & $1.508 \mathrm{e}-6$ \\
order & 2.01 & 2.02 & 2.07 & 2.32 \\
\# of iterations & 3 & 3 & 3 & 2 & 2 \\
\hline \multicolumn{5}{c}{$D_{i}=0.1, \zeta_{i}=10, i=1,2$} \\
\hline error & $1.794 \mathrm{e}-4$ & $6.097 \mathrm{e}-5$ & $1.668 \mathrm{e}-5$ & $4.077 \mathrm{e}-6$ & $8.214 \mathrm{e}-7$ \\
order & 1.56 & 1.87 & 2.03 & 2.31 \\
\# of iterations & 3 & 3 & 2 & 2 & 2 \\
\hline
\end{tabular}


TABLE 2. Numerical results for Example 5.1 with $\tau=h$.

\begin{tabular}{cccccc}
\hline$N$ & 32 & 64 & 128 & 256 & 512 \\
\hline \multicolumn{5}{c}{$D_{i}=1, \zeta_{i}=2, i=1,2$} \\
error & $6.672 \mathrm{e}-5$ & $3.148 \mathrm{e}-5$ & $1.470 \mathrm{e}-5$ & $6.345 \mathrm{e}-6$ & $2.120 \mathrm{e}-6$ \\
order & 1.08 & 1.10 & 1.21 & 1.58 & \\
\# of iterations & 3 & 3 & 3 & 3 & 3 \\
\hline \multicolumn{5}{c}{$D_{i}=0.1, \zeta_{i}=10, i=1,2$} \\
error & $1.033 \mathrm{e}-4$ & $5.659 \mathrm{e}-5$ & $2.830 \mathrm{e}-5$ & $1.258 \mathrm{e}-5$ & $4.274 \mathrm{e}-6$ \\
order & 0.87 & 1.00 & 1.17 & 1.56 & \\
\# of iterations & 3 & 3 & 3 & 2 & 2 \\
\hline
\end{tabular}

each time level for different mesh sizes $h$ and $\tau=h$. The data in the table show that the numerical solution has the first-order accuracy in the time variable. Numbers of iterations decrease as $N$ increases.

Example 5.2. In

$$
\omega=\left\{0<x_{1}<1,0<x_{2}<1\right\},
$$

we consider the second test problem:

$$
\begin{gathered}
\frac{\partial u_{1}}{\partial t}-D_{1} \Delta u_{1}+u_{1}-\alpha \int_{0}^{t} u_{2}(x, s) d s=0, \quad(x, t) \in \omega \times(0, T] \\
\frac{\partial u_{2}}{\partial t}-D_{2} \Delta u_{2}-\varphi\left(u_{1}\right)+\beta u_{2}=0 \\
\varphi\left(u_{1}\right)=\frac{u_{1}^{2}}{1+u_{1}^{2}}, \quad(x, t) \in \omega \times(0, T] \\
u_{i}(x, t)=0, \quad(x, t) \in \partial \omega \times(0, T] \\
u_{1}(x, 0)=\kappa \sin \left(\pi x_{1}\right) \sin \left(\pi x_{2}\right) \\
u_{2}(x, 0)=\varphi\left(u_{1}(x, 0)\right), \quad x \in \bar{\omega}
\end{gathered}
$$

where $D_{i}, i=1,2, \alpha, \beta$ and $\kappa$ are positive constants. For this test problem, we have

$$
f_{1}=u_{1}, \quad f_{2}=-\varphi\left(u_{1}\right)+\beta u_{2}, \quad g_{1}^{*}=-\alpha u_{2}, \quad g_{2}^{*}=0,
$$

where, for $u_{i} \geq 0, i=1,2$,

$$
\frac{\partial f_{1}}{\partial u_{1}}=1, \quad \frac{\partial f_{1}}{\partial u_{2}}=0,
$$




$$
\begin{gathered}
-\frac{\partial f_{2}}{\partial u_{1}}=\frac{2 u_{1}}{\left(1+u_{1}^{2}\right)^{2}} \geq 0, \quad \frac{\partial f_{2}}{\partial u_{2}}=\beta>0, \\
-\frac{\partial g_{1}^{*}}{\partial u_{1}}=0, \quad-\frac{\partial g_{1}^{*}}{\partial u_{2}}=\alpha, \quad \frac{\partial g_{2}^{*}}{\partial u_{i}}=0, \quad i=1,2 .
\end{gathered}
$$

From this, we choose $c_{1}=1$ and $c_{2}=\beta$ in the monotone iterative method (3.3).

The conditions in (4.5) hold true without any extra restrictions.

To guarantee (4.6), we assume that $M_{i}, i=1,2$, satisfies the inequalities

$$
M_{1}-\alpha M_{2} T \geq 0, \quad M_{1} \geq \kappa, \quad-\frac{M_{1}^{2}}{1+M_{1}^{2}}+\beta M_{2} \geq 0, \quad M_{2} \geq 1 .
$$

Thus, if we choose $M_{1}=\max \left(\kappa, \alpha M_{2} T\right)$ and $M_{2}=\max (1,1 / \beta)$, then, by Lemma 4.2, we conclude that $\widehat{U}$ and $\widetilde{U}$ from, respectively, (4.2) and (4.8), are ordered lower and upper solutions and satisfy (4.4).

We choose $D_{1}=10^{-3}, D_{2}=10^{-5}, \alpha=2.5, \beta=1$. In Table 3, for $\kappa=2$ and $\kappa=0.3$, we present the numerical error based on lower solutions as in Table 1, the order of the numerical error and numbers of monotone iterations on each time level for different mesh sizes $h$ and $\tau=h^{2}$. The data in the table indicate that the numerical solution has the second-order accuracy in the space variables, and numbers of iterations do not increase as $N$ increases.

In Table 4, for the same two sets of parameters as in Table 3, we present the numerical error based on lower solutions as in Table 1, the

TABLE 3. Numerical results for Example 5.2 with $\tau=h^{2}$.

\begin{tabular}{cccccc}
\hline$N$ & 4 & 8 & 16 & 32 & 64 \\
\hline \multicolumn{6}{c}{$\kappa=2$} \\
error & $2.328 \mathrm{e}-2$ & $5.920 \mathrm{e}-3$ & $1.470 \mathrm{e}-3$ & $3.505 \mathrm{e}-4$ & $7.013 \mathrm{e}-5$ \\
order & 1.98 & 2.01 & 2.07 & 2.32 & \\
\# of iterations & 4 & 3 & 3 & 3 & 3 \\
\hline \multicolumn{6}{c}{$\kappa=0.3$} \\
error & $3.230 \mathrm{e}-3$ & $8.231 \mathrm{e}-4$ & $2.052 \mathrm{e}-4$ & $4.973 \mathrm{e}-5$ & $9.695 \mathrm{e}-6$ \\
order & 1.97 & 2.00 & 2.04 & 2.36 & \\
\# of iterations & 3 & 3 & 3 & 3 & 2 \\
\hline
\end{tabular}


TABLE 4. Numerical results for Example 5.2 with $\tau=h$.

\begin{tabular}{cccccc}
\hline$N$ & 32 & 64 & 128 & 256 & 512 \\
\hline \multicolumn{6}{c}{$\kappa=2$} \\
error & $1.130 \mathrm{e}-2$ & $5.503 \mathrm{e}-3$ & $2.576 \mathrm{e}-3$ & $1.106 \mathrm{e}-3$ & $3.689 \mathrm{e}-4$ \\
order & 1.04 & 1.10 & 1.21 & 1.58 & \\
\# of iterations & 4 & 3 & 3 & 3 & 3 \\
\hline \multicolumn{6}{c}{$\kappa=0.3$} \\
\hline error & $1.553 \mathrm{e}-3$ & $7.566 \mathrm{e}-4$ & $3.542 \mathrm{e}-4$ & $1.520 \mathrm{e}-4$ & $5.072 \mathrm{e}-5$ \\
order & 1.04 & 1.09 & 1.22 & 1.58 & \\
\# of iterations & 3 & 3 & 3 & 3 & 3 \\
\hline
\end{tabular}

order of the numerical error and numbers of monotone iterations on each time level for different mesh sizes $h$ and $\tau=h$. The data in the table show that the numerical solution has the first-order accuracy in the time variable. Numbers of iterations do not increase as $N$ increases.

Example 5.3. In

$$
\omega=\left\{0<x_{1}<1,0<x_{2}<1\right\},
$$

we consider the third test problem which arises in population dynamics $[5]$ :

$$
\begin{gathered}
\frac{\partial u_{1}}{\partial t}-\Delta u_{1}+u_{1}\left(1-u_{2}\right)-\int_{0}^{t} \theta e^{-\theta(t-s)} u_{1}(x, s) d s=0, \\
\frac{\partial u_{2}}{\partial t}-\Delta u_{2}-u_{2}\left(u_{1}-1\right)=0, \\
(x, t) \in \omega \times(0, T], \quad u_{i}(x, t)=0, \quad(x, t) \in \partial \omega \times(0, T], \\
u_{i}(x, 0)=\sin \left(\pi x_{1}\right) \sin \left(\pi x_{2}\right), \quad x \in \bar{\omega}, \quad i=1,2,
\end{gathered}
$$

where $u_{1}$ and $u_{2}$ are, respectively, prey and predator population densities, and $\theta$ is a positive constant. The integral term represents competition for resources. For this test problem, we have

$$
\begin{array}{ll}
f_{1}=u_{1}\left(1-u_{2}\right), & f_{2}=-u_{2}\left(u_{1}-1\right), \\
g_{1}^{*}=\theta e^{-\theta(t-s)} u_{1}(x, s), & g_{2}^{*}=0 .
\end{array}
$$

where, for $u_{i} \geq 0, i=1,2$,

$$
\frac{\partial f_{1}}{\partial u_{1}}=1-u_{2}, \quad-\frac{\partial f_{1}}{\partial u_{2}=u_{1}},
$$




$$
\begin{aligned}
-\frac{\partial f_{2}}{\partial u_{1}} & =u_{2}, & & \frac{\partial f_{2}}{\partial u_{2}}=-\left(u_{1}-1\right), \\
-\frac{\partial g_{1}^{*}}{\partial u_{1}} & =\theta e^{-\theta(t-s)}, & & \frac{\partial g_{1}^{*}}{\partial u_{2}}=0, \\
\frac{\partial g_{2}^{*}}{\partial u_{i}} & =0, \quad i=1,2 . & &
\end{aligned}
$$

From this, we choose $c_{1}=1, c_{2}=1$, in the monotone iterative method (3.3). The conditions in (4.1) hold true with $\chi_{i}=0, i=1,2, \nu_{1}=\theta$ and $\nu_{2}=0$. Thus, the functions $\widehat{U}_{i}\left(p, t_{k}\right), i=1,2$, from (4.2), are lower solutions.

In Table 5 , for $\theta=1$ and $\theta=10$, we present the numerical error based on lower solutions as in Table 1, the order of the numerical error and numbers of monotone iterations on each time level for different

TABLE 5. Numerical results for Example 5.3 with $\tau=h^{2}$.

\begin{tabular}{cccccc}
\hline$N$ & 4 & 8 & 16 & 32 & 64 \\
\hline error & $2.062 \mathrm{e}-2$ & $5.238 \mathrm{e}-3$ & $1.299 \mathrm{e}-3$ & $3.099 \mathrm{e}-4$ & $6.195 \mathrm{e}-5$ \\
order & 1.98 & 2.01 & 2.07 & 2.32 & \\
\# of iterations & 4 & 3 & 3 & 3 & 2 \\
\hline \multicolumn{6}{c}{$\theta=10$} \\
\hline error & $6.831 \mathrm{e}-2$ & $1.692 \mathrm{e}-2$ & $4.160 \mathrm{e}-3$ & $9.893 \mathrm{e}-4$ & $1.978 \mathrm{e}-4$ \\
order & 2.01 & 2.02 & 2.07 & 2.32 & \\
\# of iterations & 6 & 4 & 3 & 3 & 3 \\
\hline
\end{tabular}

TABLE 6. Numerical results for Example 5.3 with $\tau=h$.

\begin{tabular}{cccccc}
\hline$N$ & 32 & 64 & 128 & 256 & 512 \\
\hline \multicolumn{6}{c}{$\theta=1$} \\
error & $8.195 \mathrm{e}-3$ & $3.963 \mathrm{e}-3$ & $1.849 \mathrm{e}-3$ & $7.924 \mathrm{e}-4$ & $2.641 \mathrm{e}-4$ \\
order & 1.05 & 1.10 & 1.22 & 1.59 & \\
\# of iterations & 3 & 3 & 3 & 3 & 3 \\
\hline \multicolumn{6}{c}{$\theta=10$} \\
\hline error & $3.420 \mathrm{e}-2$ & $1.619 \mathrm{e}-2$ & $7.470 \mathrm{e}-3$ & $3.184 \mathrm{e}-3$ & $1.060 \mathrm{e}-3$ \\
order & 1.07 & 1.16 & 1.23 & 1.58 & \\
\# of iterations & 5 & 4 & 4 & 3 & 3 \\
\hline
\end{tabular}


mesh sizes $h$ and $\tau=h^{2}$. The data in the table indicate that the numerical solution has the second-order accuracy in the space variables, and numbers of iterations do not increase as $N$ increases.

In Table 6 , for the same values of parameter $\theta$ as in Table 5 , we present the numerical error based on lower solutions as in Table 1, the order of the numerical error and numbers of monotone iterations on each time level for different mesh sizes $h$ and $\tau=h$. The data in the table show that the numerical solution has the first-order accuracy in the time variable. Numbers of iterations do not increase as $N$ increases.

\section{REFERENCES}

1. I. Boglaev, Monotone iterates for solving coupled systems of nonlinear parabolic equations, Computing 92 (2011), 65-95.

2. Monotone iterates for solving nonlinear integro-parabolic equations of Volterra type, J. Comp. Appl. Math. 290 (2015), 224-238.

3. K.J. Chang and L.Y. Tsai, On parabolic systems of integro-differential equations, Bull. Inst. Math. Acad. Sinica 29 (2001), 193-209.

4. M. Dehghan and F. Shakeri, Solution of parabolic integro-differential equations arising in heat conduction in materials with memoty via He's variational iteration, Int. J. Numer. Meth. Biomed. Eng. 26 (2010), 705-715.

5. S.A. Gourley and N.F. Britton, A predator-prey reaction-diffusion system with nonlocal effects, J. Math. Biol. 34 (1996), 297-333.

6. G.J. Habelter and R.L. Schiffman, A finite difference method for analyzing the compression of poro-viscoelastic media, Computing 6 (1970), 342-348.

7. B.G. Pachpatte, On a nonlinear diffusion system arising in reactor dynamics, J. Math. Anal. Appl. 94 (1983), 501-508.

8. C.V. Pao, Numerical methods for coupled systems of nonlinear parabolic boundary value problems, J. Math. Anal. Appl. 151 (1990), 581-608.

9. , Nonlinear parabolic and elliptic equations, Plenum Press, New York, 1992.

10. Numerical analysis of coupled systems of nonlinear parabolic equations, SIAM J. Numer. Anal. 36 (1999), 393-416.

11. A. Samarskii, The theory of difference schemes, Marcel Dekker, New York, 2001.

12. I.H. Sloan and V. Thomee, Time distretization of an integro-differential equation of parabolic type, SIAM J. Numer. Anal. 23 (1986), 1052-1061.

13. P. Souplet, Blow-up in nonlocal reaction-diffusion equations, SIAM J. Math. Anal. 29 (1998), 1301-1334.

14. A.H. Stroud, Approximate calculation of multiple integrals, Prentice-Hall, Englewood Cliffs, NJ, 1971. 
15. Y. Yamada, Asymptotic stability for some systems of semilinear Volterra diffusion equations, J. Diff. Eq. 52 (1984), 295-326.

Institute of Fundamental Sciences, Massey University, Private Bag 11-222, Palmerston North, New Zealand

Email address: i.boglaev@massey.ac.nz 